University of Wollongong

Research Online

Faculty of Engineering and Information

Faculty of Engineering and Information

Sciences - Papers: Part B

Sciences

2018

Identification of typical building daily electricity usage profiles using Gaussian mixture model-based clustering and hierarchical clustering

Kehua Li

University of Wollongong, kl751@uowmail.edu.au

Zhenjun Ma

University of Wollongong, zhenjun@uow.edu.au

Duane A. Robinson

University of Wollongong, duane@uow.edu.au

Jun Ma

University of Wollongong, jma@uow.edu.au

Follow this and additional works at: https://ro.uow.edu.au/eispapers1

Part of the Engineering Commons, and the Science and Technology Studies Commons

Research Online is the open access institutional repository for the University of Wollongong. For further information contact the UOW Library: research-pubs@uow.edu.au 


\title{
Identification of typical building daily electricity usage profiles using Gaussian mixture model-based clustering and hierarchical clustering
}

\author{
Abstract \\ This paper presents a clustering-based strategy to identify typical daily electricity usage (TDEU) profiles \\ of multiple buildings. Different from the majority of existing clustering strategies, the proposed strategy \\ consists of two levels of clustering, i.e. intra-building clustering and inter-building clustering. The intra- \\ building clustering used a Gaussian mixture model-based clustering to identify the TDEU profiles of each \\ individual building. The inter-building clustering used an agglomerative hierarchical clustering to identify \\ the TDEU profiles of multiple buildings based on the TDEU profiles identified for each individual building \\ through intra-building clustering. The performance of this strategy was evaluated using two-year hourly \\ electricity consumption data collected from 40 university buildings. The results showed that this strategy \\ can discover useful information related to building electricity usage, including typical patterns of daily \\ electricity usage (DEU) and periodical variation of DEU. It was also shown that this proposed strategy can \\ identify additional electricity usage patterns with a less computational cost, in comparison to two single- \\ step clustering strategies including a Partitioning Around Medoids-based clustering strategy and a \\ hierarchical clustering strategy. The results obtained from this study could be potentially used to assist in \\ improving energy performance of university buildings and other types of buildings.

\section{Disciplines} \\ Engineering | Science and Technology Studies

\section{Publication Details} \\ Li, K., Ma, Z., Robinson, D. \& Ma, J. (2018). Identification of typical building daily electricity usage profiles \\ using Gaussian mixture model-based clustering and hierarchical clustering. Applied Energy, 231 331-342.
}




\title{
Identification of typical building daily electricity usage profiles
}

\section{using Gaussian mixture model-based clustering and hierarchical}

\section{clustering}

\author{
Kehua $\mathrm{Li}^{1}$, Zhenjun $\mathrm{Ma}^{1, *}$, Duane Robinson ${ }^{1}$ and Jun $\mathrm{Ma}^{2}$ \\ ${ }^{1}$ Sustainable Buildings Research Centre, University of Wollongong, 2522, Australia \\ ${ }^{2}$ SMART Infrastructure Facility, University of Wollongong, 2522, Australia \\ *zhenjun@uow.edu.au
}

Abstract: This paper presents a clustering-based strategy to identify typical daily electricity usage (TDEU) profiles of multiple buildings. Different from the majority of existing clustering strategies, the proposed strategy consists of two levels of clustering, i.e. intrabuilding clustering and inter-building clustering. The intra-building clustering used a Gaussian mixture model-based clustering to identify the TDEU profiles of each individual building. The inter-building clustering used an agglomerative hierarchical clustering to identify the TDEU profiles of multiple buildings based on the TDEU profiles identified for each individual building through intra-building clustering. The performance of this strategy was evaluated using two-year hourly electricity consumption data collected from 40 university buildings. The results showed that this strategy can discover useful information related to building electricity usage, including typical patterns of daily electricity usage (DEU) and periodical variation of DEU. It was also shown that this proposed strategy can identify additional electricity usage patterns with a less computational cost, in comparison to two single-step clustering strategies including a Partitioning Around Medoids-based clustering strategy and a hierarchical clustering strategy. The results obtained from this study could be potentially used to assist in improving energy performance of university buildings and other types of buildings. 
Keywords: Building electricity usage; data mining; Gaussian mixture model; hierarchical clustering; performance comparison

\section{Nomenclature}

C

C-index

$d_{i, j}$

dissimilarity between the observations $i$ and $j$

G

number of clusters

K

number of mixture components

$M$

distance matrix

$N$

number of observations in the whole dataset

$N_{g}$

number of observations in the $g^{\text {th }}$ cluster

$N_{W}$

number of all intra-cluster observation pairs in the whole dataset

$S_{\max }$

sum of the $N_{W}$ largest pairwise distances between observations in the

whole dataset

$S_{\min }$

sum of the $N_{W}$ smallest pairwise distances between observations in the whole dataset

$S_{W}$

sum of the pairwise distances between all intra-cluster observation pairs in the whole dataset

Greek letters

$\mu$

$\omega$

$\sigma$

$\phi$

$\psi$ mean of a mixture component

weight of a mixture component

standard deviation of a mixture component

probability density function of a mixture component

probability density function of a Gaussian mixture model 
Subscripts

$g$

the $g^{\text {th }}$ cluster

$k$

the $k^{\text {th }}$ mixture component

\section{Introduction}

Buildings consume about $40 \%$ of global primary energy and produce more than $30 \%$ of $\mathrm{CO}_{2}$ emissions [1]. To improve building energy efficiency and sustainability, various technologies and solutions such as desiccant cooling [2], renewable energy integration [3], phase change materials [4], optimal control and advanced design [5] have been investigated.

Identification of building typical energy usage profiles has also been considered as a promising way to assist in understanding building energy consumption characteristics and helping the development of effective strategies to improve building energy efficiency [6]. Building energy usage profile is a time series data on energy usage of the whole building over a given period [7]. Cluster analysis, as a powerful tool which can effectively group similar objects while ensuring distinction from other grouped objects [8], has been used to identify building typical load and energy usage profiles. Miller et al. [9], for instance, proposed a method called DayFilter to detect the underlying information and identify potential areas for energy savings from building performance data and sub-system metrics. In this strategy, the building daily load profiles were first transformed into character strings using Symbolic Aggregate approXimation (SAX) and the typical daily load profiles were then identified using a $k$-means clustering method. A cluster analysis strategy to identify typical building daily load profiles using a variation-focused similarity of the load profiles was presented in [6]. The performance test of this strategy based on the hourly heating energy usage data of 19 university buildings showed that the identified typical heating energy usage profiles can provide information such as the peaks and troughs of the daily heating demand, daily high 
heating demand period and daily load variations, which were less likely to be revealed by the strategies only focused on the magnitude of load profiles. A shape definition language-based symbolic transformation technique was used to enhance the clustering result in terms of identifying the variations in the building energy usage [10]. Capozzoli et al. [11] proposed a general framework for identification and analysis of typical energy usage profiles, in which a hierarchical clustering with Ward's linkage method was first implemented to group the energy usage profiles. Two clustering validation indices were then used to identify the optimal number of partitions. Pan et al. [12] analysed the influence of the occupancy behaviour on the electricity load patterns of residential buildings using a $k$-means clustering algorithm. It was shown that the poor or elderly families tended to have a significant load shifting towards weekends while the rich and young families tended to have a fluctuating daily electricity usage curve. A clustering strategy based on a $k$-shape algorithm was proposed in [13] to identify building typical energy usage profiles. The hourly and weekly energy usage data from ten buildings were used to validate this strategy. It was shown that this strategy was effective in detecting building energy usage patterns and improving the forecasting accuracy of the Support Vector Regression (SVR) model. Multiple clustering algorithms such as $k$ means, fuzzy c-means, Self-Organizing Map (SOM), Partitioning around medoids (PAM) and hierarchical clustering have been employed to identify typical energy usage profiles of buildings. To evaluate the performance of different clustering algorithms, Panapakidis et al. [14] employed eight clustering algorithms to identify typical daily electricity usage data of 27 buildings in a university campus. A combination of SOM and $k$-means++ showed a better performance over the other clustering algorithms in terms of the clustering error. McLoughlin et al. [15] proposed a clustering-based strategy for creating representative electricity load profiles of residential buildings in Ireland. The $k$-means, $k$-medoids and SOM were used as the clustering techniques to characterize the diurnal, intra-daily and seasonal variations of 
domestic electricity demand. Tardioli et al. [16] proposed a clustering-based approach to identifying representative buildings and building groups in urban-scale datasets which contained the information about building geometries, functions and heating \& domestic hot water consumption. A total of 67 clusters were identified from 13,614 buildings in a city using $k$-means, hierarchical clustering and PAM algorithms. It is worthwhile to note that nine validation indices were employed in this study to determine the optimal clustering result. The performance evaluation of the clustering algorithms in the aforementioned studies was mainly focused on the improvement of clustering results while the computational cost of these strategies was rarely considered.

The conventional methods become less competent for identification of typical energy usage profiles from large datasets $[17,18]$. Many alternative methods have been used to deal with this problem. For instance, in a number of studies, large datasets were divided into small groups based on seasons or days of the week before performing cluster analysis. In Rhodes’ study [19], a $k$-means clustering method was used to find seasonal groups of the residential electricity usage from 103 residential buildings. The daily electricity usage (DEU) profiles of each building were first categorized into four groups according to the seasons. The means of each group were then calculated as the inputs for cluster analysis. do Carmo and Christensen [20] used a $k$-means clustering method to identify typical space heating profiles of singlefamily detached homes. One-year hourly data from 139 buildings were first categorized into groups based on weekdays and weekends as well as the intensity of building heating demand before segmentation and clustering. The results were further analyzed to investigate the correlation between household characteristics and space heating profiles using binary regression analysis. In some studies, the energy usage profiles in certain periods were aggregated before clustering. For instance, Fernandes [21] proposed a method to identify the typical natural gas consumption profiles of residential buildings. The daily gas consumption 
profiles from 1,430 households over 243 days were clustered using fuzzy c-means algorithm. In addition, sampling techniques such as random sampling and stratified sampling have also been considered as useful dimensionality reduction techniques to reduce the computational cost [22]. The above methods can save the computational cost of cluster analysis [8, 23]. However, they also considerably reduced the resolution of the input data and some meaningful information such as the variation of daily energy usage profiles based on the days of a week, seasons or holiday timetables may also be discarded.

This paper presents a new clustering-based strategy to identify typical daily electricity usage (TDEU) profiles of multiple university buildings. Different from the majority of existing studies using a single-step clustering, the TDEU profiles in the proposed strategy were identified using a two-step clustering process (i.e. intra-building clustering and interbuilding clustering) and the computational cost was a key focus during the development of this strategy. It is noted that a two-stage clustering structure was used in an early study [24] to classify electricity customers that share similar electricity usage patterns while the computational cost was not a focus. In the intra-building clustering, a Gaussian mixture model (GMM) based method was selected and used to identify the TDEU profiles of each individual building. In the inter-building clustering, an agglomerative hierarchical clustering was used to further identify the TDEU profiles of multiple buildings based on the TDEU profiles identified for each individual building. The performance of the proposed strategy was evaluated using two-year hourly building electricity usage data collected from 40 university buildings. A comparison of this proposed strategy with two other single-step clustering strategies was also performed.

\section{Strategy development}

\subsection{Outline of the proposed strategy}


The outline of the proposed clustering-based strategy to identify the TDEU profiles of multiple university buildings is presented in Fig. 1. The strategy consists of four steps, including data collection, intra-building clustering, inter-building clustering and the visualization and interpretation of the results.

The first step is to collect building electricity usage data. The intra-building clustering is then used in the second step to identify the TDEU profiles of each individual building and remove the outliers. In this step, the time series building electricity usage data were first converted into the hourly electricity usage per unit floor area and segmented into DEU profiles. After removal of the DEU profiles with missing data, a Multi-Dimensional Scaling (MDS) method was used to reduce the dimensionality of DEU profiles to enhance the computational efficiency of cluster analysis (details are provided in Section 2.2). A Gaussian mixture model (GMM) based cluster analysis was further used to cluster the DEU profiles so that the profiles in the same group are similar with each other but are different from those in other groups. In this process, the outliers were also identified and removed. The median of all DEU profiles in a cluster was then considered as the TDEU profile of that cluster. GMM was selected as the clustering method based on the comparison with other clustering algorithms as presented in Appendix and also because the building DEU profiles projected in a scatterplot can be seen as a linear superposition of several elongated ellipses with outliers and GMM can perform well under this scenario [8]. The details of GMM and GMM-based cluster analysis are introduced in Sections 2.3 and 2.4, respectively.

All the TDEU profiles identified for each individual building through intra-building clustering were then used as the input of the inter-building clustering. The inter-building clustering was then used to identify TDEU profiles for all buildings of concern based on the TDEU profiles identified for each individual building. In the inter-building clustering, the TDEU profiles identified for each individual building through intra-building clustering were 
normalized so that each profile had a mean value of 0 and variance of 1 [25]. The Euclidean distance between each pair of the normalized profiles was then calculated to determine the dissimilarity measure. An agglomerative hierarchical clustering technique was used to group the intra-building level TDEU profiles into clusters. The advantages of the hierarchical clustering are that the number of clusters can be determined during the clustering process and the overall process can be represented by a tree structure graph called dendrogram, which can help to visualize the cluster structure and assist in determining the optimal number of clusters [6].The TDEU profiles for multiple buildings were then determined by calculating the median value of all the DEU profiles in each cluster. The results from the cluster analysis were then visualized and interpreted to provide an overall understanding of the building energy performance and electricity usage behaviours.

\subsection{Multi-Dimensional Scaling (MDS)}

The increase in dimensionality of the input data can considerably increase the computational cost of the GMM training [26]. In this study, an MDS technique was employed to reduce the dimensionality of the input data before performing cluster analysis in order to reduce the computational cost. MDS is a dimensionality reduction technique that retains the major information in the raw data [27]. Compared to other well-known dimensionality reduction techniques such as piecewise aggregate approximation (PAA) and piecewise linear approximation (PLA) [28], MDS retains more useful information about the pairwise distance among the data points which is important for the implementation of the following cluster analysis [29]. This method has been widely used for data pre-processing and visualization of cluster analysis . To apply MDS to a p-dimensional raw dataset, each observation in the raw data was considered as a point in the $p$-dimensional space. The distance matrix $M$, containing all pairwise distances among the points, was then calculated. All points in the original pdimensional space were projected into a $q$-dimensional space $(q<p)$ so that the distance matrix 
of the points in the $q$-dimensional space $M^{\prime}$, is similar to $M$ as much as possible. The dimensionally reduced data can be reached by considering each point in the $q$-dimensional space as an observation in the $q$-dimensional dataset. The dissimilarity between $M$ and $M^{\prime}$ is measured using stress as defined in Eq. (1) [27].

$$
\text { Stress }=\sqrt{\frac{\sum_{i=1, j=1}^{N}\left(d_{i, j}^{\prime}-d_{i, j}\right)^{2}}{\sum_{\substack{i \neq j \\ i \neq j=1 \\ i \neq j}}^{N} d_{i, j}^{2}}}
$$

where $d_{i, j}$ and $d^{\prime}{ }_{i, j}$ denote the distances between the $i^{\text {th }}$ and $j^{\text {th }}$ points in $M$ and $M^{\prime}$, respectively.

The detailed procedure of MDS can be found in [31]. In this study, MDS was used to transform the 24-dimensional DEU profiles of each building into two-dimensional data for GMM-based clustering. Each DEU profile consists of 24 data points corresponding to 24 hours of a day, namely 24 dimensions. How to transform the data from 24 dimensions to two dimensions will be demonstrated in Section 3.2. Euclidean distance was employed as the distance measure to calculate the pairwise distances.

\subsection{Gaussian mixture model (GMM)}

A GMM is a probabilistic model to represent a dataset with a weighted combination of several normal distributions called mixture components [32]. A GMM $\psi$ with $K$ mixture components can be described as Eq. (2) [32].

$$
\left\{\begin{array}{c}
\psi(x)=\sum_{k=1}^{K} \omega_{k} \phi\left(x \mid \mu_{k}, \sigma_{k}\right) \\
\phi\left(x \mid \mu_{k}, \sigma_{k}\right)=\frac{1}{\sigma_{k} \sqrt{2 \pi}} \exp \left(-\frac{\left(x-\mu_{k}\right)^{2}}{2 \sigma_{k}^{2}}\right) \\
\sum_{k=1}^{K} \omega_{k}=1
\end{array}\right.
$$


where $\phi$ is the Gaussian probability density function, and $\omega_{k}, \mu_{k}$ and $\sigma_{k}$ are the weight, mean and standard deviation of the $k^{\text {th }}$ mixture component, respectively.

Given a dataset and $K$ value, a GMM fitting is to estimate the values of the parameters $\omega_{k}, \mu_{k}$ and $\sigma_{k}$ to ensure that the GMM has the maximum likelihood. The ExpectationMaximisation (EM) algorithm is commonly used to fit GMM and this algorithm makes an initial guess for the parameters and then iteratively improves the estimates [8]. The implementation of EM consists of three steps, including initialisation, expectation step and maximisation step [33]. An initial set of the model parameters was first randomly selected. An iteration of expectation step and maximization step was then conducted to improve the estimation of the model parameters. In the expectation step, each observation was assigned to one of the mixture components which assigns the highest probability to this observation. Given the observations assigned in each mixture component in the expectation step, the parameter of each mixture component was updated in the maximisation step based on the location of the observations assigned to this mixture component. The iteration of expectation step and maximization step will terminate when the updated parameters of all mixture components do not change further. In this study, a modified EM algorithm proposed by Banfield and Raftery [34] was employed to fit the GMMs. This algorithm can identify and remove the observations that have a low probability in any mixture component, which were considered as the outliers in order to improve the robustness of the clustering results.

\subsection{Gaussian mixture model-based clustering}

GMM-based clustering, as shown in Fig. 2a), uses the feature of GMM to group multiple observations in a dataset into different clusters [34]. To conduct GMM-based clustering, a GMM with $K$ mixture components was first fitted with the two-dimensional input data transformed via MDS, in which each observation denotes an original DEU profile. Once the GMM has been fitted, the observations belonging to the same mixture component were 
considered as in the same cluster. The median of the corresponding original DEU profiles in the same cluster was then considered as the TDEU profile of this cluster.

A key task involved in the GMM-based clustering is to determine the optimal number of the mixture components, $K$. Bayesian Information Criterion (BIC) [35], as one of the most widely used tools for statistical model selection, was used for this purpose. Assuming that the input data was originally generated according to an unknown GMM (i.e. $\psi_{\text {true }}$ ) and the GMM fitted using the generated input data (i.e. $\left.\psi_{\text {candidate }}\right)$, BIC is then used to measure the

difference between $\psi_{\text {true }}$ and $\psi_{\text {candidate }}[36,37]$. Hence, the GMM with an optimal $K$ value has the lowest BIC. For each building, the $K$ value which can minimise BIC was used as the optimal $K$ number for GMM fitting. In general, identifying optimal $K$ in a big range is computationally intensive [6]. The previous studies showed that the number of TDEU profiles for single buildings generally varied from 2 to 8 [9, 10, 13, 19, 38, 39]. The optimal $K$ value in the intra-building clustering used in this study was determined based on a range of 214.

\subsection{Agglomerative hierarchical clustering}

The inter-building clustering was achieved by using an agglomerative hierarchical clustering. The agglomerative hierarchical clustering is a bottom-up clustering strategy, which starts with treating each observation as a separate cluster and then merges the atomic clusters into larger clusters until all objects are in a single cluster [8]. Dissimilarity measure and linkage criteria are the two important components of a hierarchical clustering algorithm. In this study, Euclidean Distance was used as the dissimilarity measure and Ward's method was used as the linkage criterion. Compare with other commonly used linkage criteria such as single linkage, complete linkage and group average linkage, Ward's method showed a better performance in terms of clustering accuracy [40] and computational cost [41]. In Ward's method, two clusters should be merged if the merge can minimize the increase in the sum of 
squared error [42]. In this study, C-Index was used to validate the result of the hierarchical clustering and determine the optimal number of clusters. C-Index is a measure of how similar each member is to its own cluster compared to other clusters [43]. A lower C-Index means a better clustering result. The optimal number of clusters $G$ was determined based on the lowest C-Index within a defined range of the number of clusters. C-Index $C$ is defined as in Eq. (3) [43].

$$
\left\{\begin{array}{c}
C=\frac{S_{W}-S_{\min }}{S_{\max }-S_{\min }} \\
S_{W}=\sum_{g}^{G} \sum_{\substack{i=1, j=1 \\
i \neq j}}^{N_{g}} d_{i, j} \\
N_{W}=\sum_{g}^{G} \frac{N_{g}\left(N_{g}-1\right)}{2}
\end{array}\right.
$$

where $N_{W}$ is the number of the intra-cluster observation pairs in the whole dataset, $S_{\min }$ is the sum of the $N_{W}$ smallest distances between all the pairs of the observations in the whole dataset, and $S_{\max }$ is the sum of the $N_{W}$ largest distances between all the pairs of the observations in the whole dataset. Fig. 2b) illustrates the procedure of the agglomerative hierarchical clustering algorithm used in this study.

\section{Performance test and evaluation of the proposed strategy}

In this study, the proposed strategy was implemented in $R$ [44] while the GMM-based clustering and agglomerative hierarchical clustering were implemented using $R$ package mclust [45] and cluster [46], respectively. The majority of the figures presented was generated using $R$ package ggplot2 [47].

\subsection{Description of the case study buildings}

The performance of the proposed strategy was tested using the hourly electricity usage data collected from 40 buildings at the University of Wollongong (Fig. 3) from 2014 to 2015. The functions of these 40 buildings varied from offices, education rooms and laboratories to 
sports centres, student accommodations and common areas, as summarised in Table 1. Fig. 4 shows the average hourly building electricity usage against the building floor area. It can be seen that the mean hourly electricity usage among these 40 buildings varied considerably from less than $0.001 \mathrm{kWh} / \mathrm{m}^{2}$ (Building \#39) to more than $0.045 \mathrm{kWh} / \mathrm{m}^{2}$ (Building \#32). Even for the buildings with similar functions, a large difference was also observed. For instance, Building \#14 and Building \#20 are mainly used for offices with the similar floor area. However, the mean hourly electricity usage of Building \#20 was almost 5 times higher than that of Building \#14.

\subsection{Results of the intra-building clustering}

The time series electricity usage data of each building were first transformed into the electricity usage per square meter and then segmented into the DEU profiles. An illustration of the data segmentation is shown in Fig. 5. The hourly electricity usage data of a whole week (Fig. 5a) were segmented into 7 DEU profiles as shown in Fig. 5b). Each DEU profile consists of 24 data points (i.e. 24 dimensions) corresponding to 24 hours of a day. The bold curves in Fig. 5a) and Fig. 5b) represented the same set of the data points. In this process, the DEU profiles with missing data were removed in the following analysis. After the completion of the data pre-processing, an average of 695 DEU profiles remained for each building.

The DEU profiles of each building were then projected into a two-dimensional coordinate system using the MDS technique. A GMM for each building was then fitted in the twodimensional coordinate system to identify the TDEU profiles and outliers. The number of clusters (i.e. the number of TDEU profiles) identified for each building through intra-building clustering is summarised in Table 1. It is noted that the dimensionally reduced data were only used for visualization and clustering processing, and the output of intra-building clustering was still in 24-dimensions. It can be seen that there were twelve buildings that had three 
TDEU profiles. The buildings with two to three TDEU profiles accounted for $50 \%$ of all 40 buildings investigated. Most of the buildings had no more than six TDEU profiles.

Fig. 6 illustrates the results from the GMM-based clustering for three selected buildings (i.e. Building \#16, Building \#33 and Building \#24), where the clusters and outliers were identified. The black circles indicated the location and covariance of the clusters, while the red symbols represented the observations which were far away from any mixture components (i.e. clusters) and from each other, and subsequently identified as outliers.

It can be seen that the majority of the clusters (i.e. black circles) identified were in elongated ellipses. This means that the commonly used $k$-means clustering algorithms may not work well for this case as it has difficulty in identifying clusters with non-spherical shapes or with widely different sizes or densities [8]. The identified TDEU profiles for these three selected buildings are presented as follows.

\subsubsection{TDEU profiles of Building \#16}

Fig. 7a) illustrates the inter-building clustering result of Building \#16, which is an office building. The grey curves in the figure represented the TDEU profiles identified while the colored curves were all corresponding DEU profiles in that cluster. It can be seen that there were two TDEU profiles with 220 and 467 DEU profiles respectively that were identified for this building and 28 DEU profile were considered as outliers. It can also be seen that there was a clear high electricity consumption period (8:00 to 16:00) during the working hours and a low electricity consumption period during the rest of the day in TDEU profile 2, while such information cannot be observed in Cluster 1. For the DEU profiles identified as the outliers, the electricity usage profiles varied significantly during the daytime, which were clearly different from the DEU profiles in the two clusters.

Fig. 7b) shows the distribution of the TDEU profiles in a calendar review, in which the white blocks represented the days with the missing data that were removed during the data 
pre-processing. It is shown that TDEU profile 2 represented the DEU profiles of the weekdays while TDEU profile 1 mainly appeared on weekends and some public holidays such as Australia Day, Easter and Labour Day.

\subsubsection{TDEU profiles of Building \#33}

Fig. 8 presents the clustering result of Building \#33, which is a student accommodation. There were three TDEU profiles identified for this building and 14 DEU profiles were considered as the outliers. TDEU profiles 1 and 3 shared a similar shape with two peak demands occurring at around 10:00 and 21:00, respectively. However, the electricity usage of TDEU profile 1 was considerably higher than that of TDEU profile 3. This mainly occurred in winter, refer to Fig. 8b, and was probably due to the increased hot water requirement and space heating requirement. TDEU profile 2 represented the electricity usage behaviour during the summer holidays (late November to late February). The electricity consumption during this period was relatively small and stable as there were only a limited number of students occupying the building. The calendar view showed that the distribution of the DEU profiles was mainly influenced by the summer holidays and seasonal variations. It is interesting to note that the first two weeks in January 2014 were mainly dominated with the TDEU profiles 1 and 3 with high energy consumption while those in 2015 were mainly dominated with the TDEU profile 2, which may be worthwhile to further investigate.

\subsubsection{TDEU profiles of Building \#24}

Building \#24 is a multi-functional building mainly used for offices and educational rooms. The clustering results are presented in Fig. 9. It can be seen that there were four TDEU profiles identified for this building. TDEU profile 2 represented the summer weekdays and the electricity usage significantly increased and decreased at 6:00 and 17:00, respectively. TDEU profile 4 represented the electricity usage behaviour in winter weekdays. TDEU profiles 1 and 3 showed different trends and represented the DEU profiles in the summer 
weekends and winter weekends, respectively. From Fig. 9b), it can be seen that the distribution of the DEU profiles of this building was mainly influenced by the seasonal variations and days of the week.

\subsubsection{Variation of the intra-building TDEU profiles}

According to the distribution of the TDEU profiles of each building, the buildings were classified into four groups. In Group 1, the buildings had only one TDEU profile during the weekdays. In Group 2, there were more than one TDEU profile during the weekdays which mainly presented the DEU profiles during the winter and summer periods. In Group 3, there was more than one TDEU profile but they represented the electricity usage during holidays, session breaks and session time. In the last group, there was not a clear pattern for the distribution of the TDEU profiles. The percentage of the number of the buildings in each group to the total number of the buildings with the same function is presented in Fig. 10. For instance, two sports centres were considered in this study. One of them was classified into Group 2 and the other was in the Group 4. Their proportions were therefore 50\% each. The buildings with office functions tended to have a high diversity in terms of the distribution of the TDEU profiles. It was interesting to note that all laboratory buildings had no obvious pattern in terms of the distribution of the TDEU profiles, which reflected the complexity and large variation in electricity usage in such buildings.

\subsection{Results of the inter-building clustering}

Through the intra-building clustering analysis, a total of 165 24-dimensional TDEU profiles were identified for all 40 buildings (Table 1). These profiles were then clustered with the agglomerative hierarchical clustering algorithm. Since several previous studies reported that the number of the TDEU profiles identified for multiple buildings was varied from 5 to $12[6,9,15,20,48]$, the search range of the cluster number used in this study was from 3 to 15. Fig. 11a) shows the variations of the value of C-Index with the increase of the cluster 
number. It can be observed that C-Index reached the minimum value when the cluster number was nine, which was therefore chosen as the optimal number of the clusters in the interbuilding clustering. Fig. 11b) illustrates how hierarchical clustering formed a tree structure with the TDEU profiles. A total of nine clusters were identified for 40 buildings based on the TDEU profiles identified for each individual building.

Fig. 12 shows the 24-dimensional TDEU profiles identified for all 40 buildings through inter-building clustering. The TDEU profiles presented in Fig. 12a) were determined based on the average of the TDEU profiles identified through intra-building clustering for individual buildings belonging to that cluster (i.e. the TDEU profile identified through inter-building clustering), while that presented in Fig. 12b) was determined based on the average of the original DEU profiles corresponding to the TDEU profiles identified for individual buildings belonging to that cluster. The key difference between the two approaches was the use of the TDEU profiles identified through intra-building clustering or the use of original DEU profiles to determine the TDEU profiles for all buildings of concern.

It can be seen that both provided very similar results but the former one can slightly save the computational cost. In TDEU profile 3, the boundary between the working hours and nonworking hours was clear and the large electricity demand occurred between 8:00 and 17:00. TDEU profile 7 also had a clear boundary between the working hours and non-working hours but with a longer large electricity demand period (i.e. from 6:00 to 21:00) than that of TDEU profile 3. In TDEU profiles 1 and 4, the peak demand occurred at around 12:00 but the boundary between the working hour and non-working hours was unclear at late afternoon and morning, respectively. TDEU profiles 2 and 9 were mostly identified in the student accommodations. In TDEU profiles 5 and 6, the electricity consumption during non-working hours was considerably higher than that during the working hours. TDEU profile 8 did not show a clear boundary between the working hours and non-working hours. 
The original DEU profiles of each individual building belonging to each inter-building level clusters (i.e. TDEU profiles) were then used to determine the distribution of the interbuilding level clusters in each building and the results are presented in Fig. 13.

It can be seen that TDEU profile 1 was dominated in the buildings used as laboratories or office/laboratories. TDEU profile 7 mainly occurred in the sports centres and two buildings with a common area and one office building. It is interesting to note that, around $50 \%$ of the time, Building \#9 was operated with the TDEU profile 5, which rarely occurred in other buildings with similar functions, and is worthwhile to further investigate.

\section{Comparison between the proposed strategy with two single-step clustering strategies}

In this section, the performance of the proposed strategy was compared with two singlestep clustering strategies, in which only inter-building clustering was employed. One was a Partitioning Around Medoids (PAM) clustering-based strategy used in [6] and the other was a hierarchical clustering-based strategy used in [39].

In both single-step clustering strategies, the Generalized Extreme Studentised Deviate (GESD) test method was used to identify and remove the outliers from the raw dataset. The DEU profiles were standardized to zero mean and one standard deviation for clustering. Data segmentation was then used to transform the data into 24-hour segments in order to form DEU profiles and the segments with a small difference between the daily maximum and minimum energy usage were discarded (i.e. $5.0 \%$ out of the total). The only difference between the two single-step strategies is the method used for clustering. In the PAM clustering method, a Pearson Correlation Coefficient (PCC)-based distance [6] was used to determine the dissimilarity matrix, and the PAM clustering algorithm was used to cluster the DEU profiles. In the hierarchical clustering strategy, the Euclidean distance was used to determine the dissimilarity and the hierarchical clustering strategy was used to cluster the DEU profiles. Table 2 summarizes the difference between the two single-step clustering 
strategies and the proposed strategy as well as their computational cost, which were obtained based on the same computer.

Fig. 14 presents the clustering results and the identified TDEU profiles using the PAMbased strategy. It can be seen that most of the TDEU profiles identified were similar to that identified by the proposed strategy (Fig. 12). However, there was a relatively large variation in the TDEU profiles identified by the proposed strategy and those identified by the PAMbased clustering were relatively flat. The computational cost of the PAM-based strategy to cluster the DEU profiles was more than 30 times of that used by the proposed strategy.

Fig. 15 presents the clustering results and the identified TDEU profiles by using the hierarchical clustering-based strategy. In comparison to the results obtained from the proposed strategy, a lot of interesting information such as unique DEU profiles of the student accommodation and sports centres (i.e. TDEU profiles 9 and 7 in Fig. 12 respectively) cannot be reflected in the TDEU profiles identified by the hierarchical clustering-based strategy. The computational cost of the hierarchical clustering-based strategy was around 16.5 times higher than the proposed strategy. The above comparison demonstrated the effectiveness of the proposed strategy in terms of the computational cost required and discovering the meaningful information from the large dataset.

\section{Conclusion}

This paper presented a fast clustering-based strategy to identify TDEU profiles of multiple university buildings. In this strategy, the TDEU profiles of each building were first identified using Gaussian mixture model-based clustering. The identified TDEU profiles for all individual buildings were then further clustered using a hierarchical clustering with Euclidean distance as the dissimilarity measure.

The performance of this strategy was evaluated using two-year hourly electricity usage data collected from 40 buildings in a university campus in Australia. The results showed that 
this strategy can discover useful information related to the electricity usage behaviours of multiple buildings. In comparison to a Partitioning Around Medoids-based clustering strategy and a hierarchical clustering strategy, the computational cost of the proposed strategy decreased by $97 \%$ and 94\%, respectively. Abnormal electricity consumption patterns were also discovered and analysed.

The building electricity usage patterns identified by the proposed strategy can be used to group buildings that share similar electricity usage behaviours and further to assist in the decision making for building energy efficient retrofits and performance enhancement. The information discovered can also be useful for developing advanced building energy management and fault detection \& diagnosis strategies. The proposed strategy can be potentially adapted and used for energy planning of campus buildings.

\section{Appendix}

In this study, the GMM-based clustering algorithm for intra-building clustering was selected based on the comparison with seven other clustering algorithms. These included a $k$ means algorithm, a PAM algorithm, a SOM clustering algorithm and four agglomerative hierarchical clustering (AHC) algorithms using complete linkage, single linkage, average linkage and Ward's method criterion, respectively. Silhouette index, Dunn index and DaviesBouldin index (DB index) were employed to evaluate the clustering results. The details about Silhouette index, Dunn index and Davies-Bouldin index (DB index) can be found in [6, 16, 21]. A higher value of Silhouette index and Dunn index indicates a better clustering result while a lower value of DB index means a better clustering result.

Building \#16, presented in Section 3.1, was chosen for this comparison. Before clustering, the GESD test method was used to identify and remove the outliers from the raw dataset and the time series building electricity usage data were converted into the hourly electricity usage 
per unit floor area and segmented into DEU profiles. The DEU profiles with missing data were also removed before clustering. The optimal cluster number for each algorithm was determined based on a range of 2-14. Fig. A.1 presents the clustering results of using different clustering algorithms based on their corresponding optimal cluster numbers. It can be seen that the GMM-based clustering outperformed the other clustering algorithms in terms of the three indices selected. It was therefore used for intra-building clustering in this study.

Table A.1 Comparison of the clustering results by using different clustering algorithms.

\begin{tabular}{|c|c|c|c|c|c|c|}
\hline \multirow{2}{*}{ Clustering algorithms } & \multicolumn{2}{|c|}{ Silhouette index } & \multicolumn{2}{c|}{ Dunn index } & \multicolumn{2}{c|}{ DB index } \\
\cline { 2 - 7 } & $\begin{array}{c}\text { Optimal } \\
\text { cluster } \\
\text { number }\end{array}$ & $\begin{array}{c}\text { Maximum } \\
\text { value }\end{array}$ & $\begin{array}{c}\text { Optimal } \\
\text { cluster } \\
\text { number }\end{array}$ & $\begin{array}{c}\text { Maximum } \\
\text { value }\end{array}$ & $\begin{array}{c}\text { Optimal } \\
\text { cluster } \\
\text { number }\end{array}$ & $\begin{array}{c}\text { Minimum } \\
\text { value }\end{array}$ \\
\hline GMM & 2 & 0.719 & 2 & 0.441 & 2 & 0.391 \\
\hline k-means & 2 & 0.696 & 2 & 0.125 & 2 & 0.488 \\
\hline PAM & 2 & 0.696 & 2 & 0.125 & 2 & 0.488 \\
\hline SOM & 2 & 0.693 & 2 & 0.135 & 2 & 0.497 \\
\hline AHC with complete & 2 & 0.689 & 14 & 0.188 & 2 & 0.509 \\
\hline linkage & 2 & 0.401 & 2 & 0.333 & 8 & 0.569 \\
\hline AHC with single linkage & 2 & 0.696 & 9 & 0.279 & 8 & 0.462 \\
\hline AHC with average linkage & 2 & 0.696 & 2 & 0.183 & 2 & 0.472 \\
\hline
\end{tabular}

\section{References}

[1] Costa A, Keane MM, Torrens JI, Corry E. Building operation and energy performance: Monitoring, analysis and optimisation toolkit. Applied Energy. 2013;101:310-6.

[2] Enteria N, Yoshino H, Satake A, Mochida A, Takaki R, Yoshie R, et al. Development and construction of the novel solar thermal desiccant cooling system incorporating hot water production. Applied Energy. 2010;87:478-86.

[3] Zogou O, Stapountzis H. Energy analysis of an improved concept of integrated PV panels in an office building in central Greece. Applied Energy. 2011;88:853-66. 
[4] Ma Z, Lin W, Sohel MI. Nano-enhanced phase change materials for improved building performance. Renewable and Sustainable Energy Reviews. 2016;58:1256-68.

[5] Huang S, Ma Z, Cooper P. Optimal design of vertical ground heat exchangers by using entropy generation minimization method and genetic algorithms. Energy Conversion and Management. 2014;87:128-37.

[6] Ma Z, Yan R, Nord N. A variation focused cluster analysis strategy to identify typical daily heating load profiles of higher education buildings. Energy. 2017;134:90-102.

[7] Bicego M, Farinelli A, Grosso E, Paolini D, Ramchurn S. On the distinctiveness of the electricity load profile. Pattern Recognition. 2018;74:317-25.

[8] Tan P-N, Steinbach M, Kumar V. Introduction to data mining. Boston: Pearson Addison Wesley; 2005.

[9] Miller C, Nagy Z, Schlueter A. Automated daily pattern filtering of measured building performance data. Automation in Construction. 2015;49:1-17.

[10] Ma Z, Yan R, Li K, Nord N. Building energy performance assessment using volatility change based symbolic transformation and hierarchical clustering. Energy and Buildings. 2018;166:284-95.

[11] Capozzoli A, Piscitelli MS, Brandi S, Grassi D, Chicco G. Automated load pattern learning and anomaly detection for enhancing energy management in smart buildings. Energy. 2018;157:336-52.

[12] Pan S, Wang X, Wei Y, Zhang X, Gal C, Ren G, et al. Cluster analysis for occupantbehavior based electricity load patterns in buildings: A case study in Shanghai residences. Building Simulation: Springer; 2017. p. 889-98.

[13] Yang J, Ning C, Deb C, Zhang F, Cheong D, Lee SE, et al. k-Shape clustering algorithm for building energy usage patterns analysis and forecasting model accuracy improvement. Energy and Buildings. 2017;146:27-37. 
[14] Panapakidis IP, Papadopoulos TA, Christoforidis GC, Papagiannis GK. Pattern recognition algorithms for electricity load curve analysis of buildings. Energy and Buildings. 2014;73:137-45.

[15] McLoughlin F, Duffy A, Conlon M. A clustering approach to domestic electricity load profile characterisation using smart metering data. Applied energy. 2015;141:190-9.

[16] Tardioli G, Kerrigan R, Oates M, O'Donnell J, Finn DP. Identification of representative buildings and building groups in urban datasets using a novel pre-processing, classification, clustering and predictive modelling approach. Building and Environment. 2018;140:90-106.

[17] Aghabozorgi S, Ying Wah T, Herawan T, Jalab HA, Shaygan MA, Jalali A. A hybrid algorithm for clustering of time series data based on affinity search technique. The Scientific World Journal. 2014.

[18] Bendechache M, Kechadi M-T, Le-Khac N-A. Efficient Large Scale Clustering based on data partitioning. 2016 IEEE International Conference on Data Science and Advanced Analytics: IEEE; 2016. p. 612-21.

[19] Rhodes JD, Cole WJ, Upshaw CR, Edgar TF, Webber ME. Clustering analysis of residential electricity demand profiles. Applied Energy. 2014;135:461-71.

[20] do Carmo CMR, Christensen TH. Cluster analysis of residential heat load profiles and the role of technical and household characteristics. Energy and Buildings. 2016;125:171-80.

[21] Fernandes MP, Viegas JL, Vieira SM, Sousa JM. Analysis of residential natural gas consumers using fuzzy c-means clustering. 2016 IEEE International Conference on Fuzzy Systems: IEEE; 2016. p. 1484-91.

[22] Fan C, Xiao F, Yan C. A framework for knowledge discovery in massive building automation data and its application in building diagnostics. Automation in Construction. 2015;50:81-90. 
[23] Fu T-c. A review on time series data mining. Engineering Applications of Artificial Intelligence. 2011;24:164-81.

[24] Tsekouras GJ, Hatziargyriou ND, Dialynas EN. Two-stage pattern recognition of load curves for classification of electricity customers. IEEE Transactions on Power Systems. 2007;22:1120-8.

[25] Yan R, Ma Z, Kokogiannakis G, Zhao Y. A sensor fault detection strategy for air handling units using cluster analysis. Automation in Construction. 2016;70:77-88.

[26] Bouveyron C, Brunet-Saumard C. Model-based clustering of high-dimensional data: A review. Computational Statistics \& Data Analysis. 2014;71:52-78.

[27] Cox TF, Cox MA. Multidimensional scaling: CRC press; 2000.

[28] Hou C, Ouyang M, Xu L, Wang H. Approximate Pontryagin’s minimum principle applied to the energy management of plug-in hybrid electric vehicles. Applied Energy. 2014;115:174-89.

[29] Li R, Wang Z, Gu C, Li F, Wu H. A novel time-of-use tariff design based on Gaussian Mixture Model. Applied energy. 2016;162:1530-6.

[30] Raatikainen M, Skön J-P, Leiviskä K, Kolehmainen M. Intelligent analysis of energy consumption in school buildings. Applied Energy. 2016;165:416-29.

[31] Torgerson WS. Multidimensional scaling: I. Theory and method. Psychometrika. 1952;17:401-19.

[32] Han J, Pei J, Kamber M. Data mining: concepts and techniques: Elsevier; 2011.

[33] Figueiredo MAT, Jain AK. Unsupervised learning of finite mixture models. IEEE Transactions on pattern analysis and machine intelligence. 2002;24:381-96.

[34] Banfield JD, Raftery AE. Model-based Gaussian and non-Gaussian clustering. Biometrics. 1993:803-21. 
[35] Hsu D. Comparison of integrated clustering methods for accurate and stable prediction of building energy consumption data. Applied energy. 2015;160:153-63.

[36] Neath AA, Cavanaugh JE. The Bayesian information criterion: background, derivation, and applications. Wiley Interdisciplinary Reviews: Computational Statistics. 2012;4:199-203. [37] Schwarz G. Estimating the dimension of a model. The annals of statistics. 1978;6:461-4. [38] Capozzoli A, Piscitelli MS, Brandi S. Mining typical load profiles in buildings to support energy management in the smart city context. Energy Procedia. 2017;134:865-74.

[39] Seem JE. Pattern recognition algorithm for determining days of the week with similar energy consumption profiles. Energy and Buildings. 2005;37:127-39.

[40] Blashfield RK. Mixture model tests of cluster analysis: Accuracy of four agglomerative hierarchical methods. Psychological Bulletin. 1976;83:377-89.

[41] Murtagh F. Expected-time complexity results for hierarchic clustering algorithms which use cluster centres. Inf Process Lett. 1983;16:237-41.

[42] Ward Jr JH. Hierarchical grouping to optimize an objective function. Journal of the American statistical association. 1963;58:236-44.

[43] Desgraupes B. Clustering indices. University of Paris Ouest-Lab ModalX. 2013.

[44] R Development Core Team. R: A language and environment for statistical computing. 2013.

[45] Scrucca L, Fop M, Murphy TB, Raftery AE. mclust 5: Clustering, classification and density estimation using gaussian finite mixture models. The R journal. 2016;8:289-317.

[46] Maechler M, Rousseeuw P, Struyf A, Hubert M, Hornik K. Cluster: cluster analysis basics and extensions. R package version. 2015.

[47] Wickham H. ggplot2: elegant graphics for data analysis: Springer; 2016.

[48] Luo X, Hong T, Chen Y, Piette MA. Electric load shape benchmarking for small-and medium-sized commercial buildings. Applied Energy. 2017;204:715-25. 
Table 1 Building information and number of clusters identified through intra-building clustering.

\begin{tabular}{|c|c|c|c|c|c|}
\hline Building No. & $\begin{array}{c}\text { Main } \\
\text { functions }\end{array}$ & $\begin{array}{c}\text { Number of } \\
\text { clusters } \\
\text { identified }\end{array}$ & Building No. & $\begin{array}{c}\text { Main } \\
\text { functions }\end{array}$ & $\begin{array}{c}\text { Number of } \\
\text { clusters } \\
\text { identified }\end{array}$ \\
\hline$\# 1$ & O/L & 2 & $\# 21$ & O/E & 2 \\
\hline$\# 2$ & O/L & 3 & $\# 22$ & O/E & 8 \\
\hline$\# 3$ & O/L & 3 & $\# 23$ & O/E & 3 \\
\hline$\# 4$ & O/L & 3 & $\# 24$ & O/E & 4 \\
\hline$\# 5$ & O/L & 2 & $\# 25$ & O/E & 4 \\
\hline$\# 6$ & O/L & 3 & $\# 26$ & O/E & 2 \\
\hline$\# 7$ & O/L & 3 & $\# 27$ & E & 6 \\
\hline$\# 8$ & O/L & 3 & $\# 28$ & E & 8 \\
\hline$\# 9$ & O/L & 5 & $\# 29$ & E & 6 \\
\hline$\# 10$ & O/L & 2 & $\# 30$ & L & 3 \\
\hline$\# 11$ & O/L & 4 & $\# 31$ & L & 6 \\
\hline$\# 12$ & O/L & 3 & $\# 32$ & L & 2 \\
\hline$\# 13$ & O/L & 5 & $\# 33$ & A & 3 \\
\hline$\# 14$ & O & 3 & $\# 34$ & A & 4 \\
\hline$\# 15$ & O & 4 & $\# 35$ & C & 6 \\
\hline$\# 16$ & O & 2 & $\# 36$ & C & 4 \\
\hline$\# 17$ & O & 3 & $\# 37$ & L/C & 12 \\
\hline$\# 18$ & O & 6 & $\# 38$ & L/C & 2 \\
\hline$\# 19$ & O & 6 & $\# 39$ & S & 5 \\
\hline$\# 20$ & O & 5 & $\# 40$ & S & 5 \\
\hline Total number of TDEU profiles identified via intra-building clustering & 165 \\
\hline
\end{tabular}

O: office; E: educational room; L: laboratory; C: common area; S: sports centre; A: student accommodation.

Table 2 Differences and computational costs of three strategies.

\begin{tabular}{|c|c|c|c|c|c|c|}
\hline Strategy & $\begin{array}{l}\text { Clustering } \\
\text { algorithm }\end{array}$ & $\begin{array}{l}\text { Dissimilarity } \\
\text { measure }\end{array}$ & $\begin{array}{c}\text { Range of } \\
\text { number of } \\
\text { clusters }\end{array}$ & $\begin{array}{l}\text { Clusteri } \\
\text { ng } \\
\text { index }\end{array}$ & $\begin{array}{l}\text { Number of } \\
\text { clusters } \\
\text { identified }\end{array}$ & $\begin{array}{l}\text { Computational } \\
\text { cost (s) }\end{array}$ \\
\hline $\begin{array}{c}\text { Proposed } \\
\text { strategy }\end{array}$ & $\begin{array}{c}\text { GMM-based } \\
\text { clustering } \\
\text { (Intra-building) } \\
\& \\
\text { agglomerative } \\
\text { hierarchical } \\
\text { clustering } \\
\text { (Inter-building) }\end{array}$ & $\begin{array}{l}\text { Euclidean } \\
\text { distance }\end{array}$ & $\begin{array}{c}2 \sim 14 \\
\text { (Intra- } \\
\text { building) } \\
\& \\
3 \sim 15 \\
\text { (Inter- } \\
\text { building) }\end{array}$ & C-index & 9 & 82.87 \\
\hline $\begin{array}{l}\text { PAM-based } \\
\text { clustering }\end{array}$ & PAM & $\begin{array}{c}\text { PCC-based } \\
\text { distance }\end{array}$ & $3 \sim 15$ & $\begin{array}{l}\text { Dunn } \\
\text { index }\end{array}$ & 9 & 2775.59 \\
\hline $\begin{array}{l}\text { Hierarchical } \\
\text { clustering }\end{array}$ & $\begin{array}{l}\text { Agglomerative } \\
\text { hierarchical } \\
\text { clustering }\end{array}$ & $\begin{array}{l}\text { Euclidean } \\
\text { distance }\end{array}$ & $3 \sim 15$ & $\begin{array}{l}\text { Dunn } \\
\text { index }\end{array}$ & 4 & 1367.17 \\
\hline
\end{tabular}




\section{Figure captions}

Fig. 1. Outline of the proposed strategy.

Fig. 2. Flowcharts of the two clustering algorithms used in this study.

Fig. 3. Illustration of the university buildings concerned in this study.

Fig. 4. Mean hourly electricity usage versus the floor area of 40 buildings.

Fig. 5. An illustration of the data segmentation.

Fig. 6. A demonstration of the results from the GMM-based clustering.

Fig. 7. Visualisation of the intra-building clustering result of Building \#16.

Fig. 8. Visualisation of the intra-building clustering result of Building \#33.

Fig. 9. Visualisation of the intra-building clustering result of Building \#24.

Fig. 10. Distribution of the buildings with different functions into the defined four groups.

Fig. 11. The result of the agglomerative hierarchical clustering.

Fig. 12. TDEU profiles identified through hierarchical clustering for all 40 buildings.

Fig. 13. The distribution of TDEU profiles of 40 buildings.

Fig. 14. Clustering result of the PAM-based strategy.

Fig. 15. Clustering result of the hierarchical clustering-based strategy. 


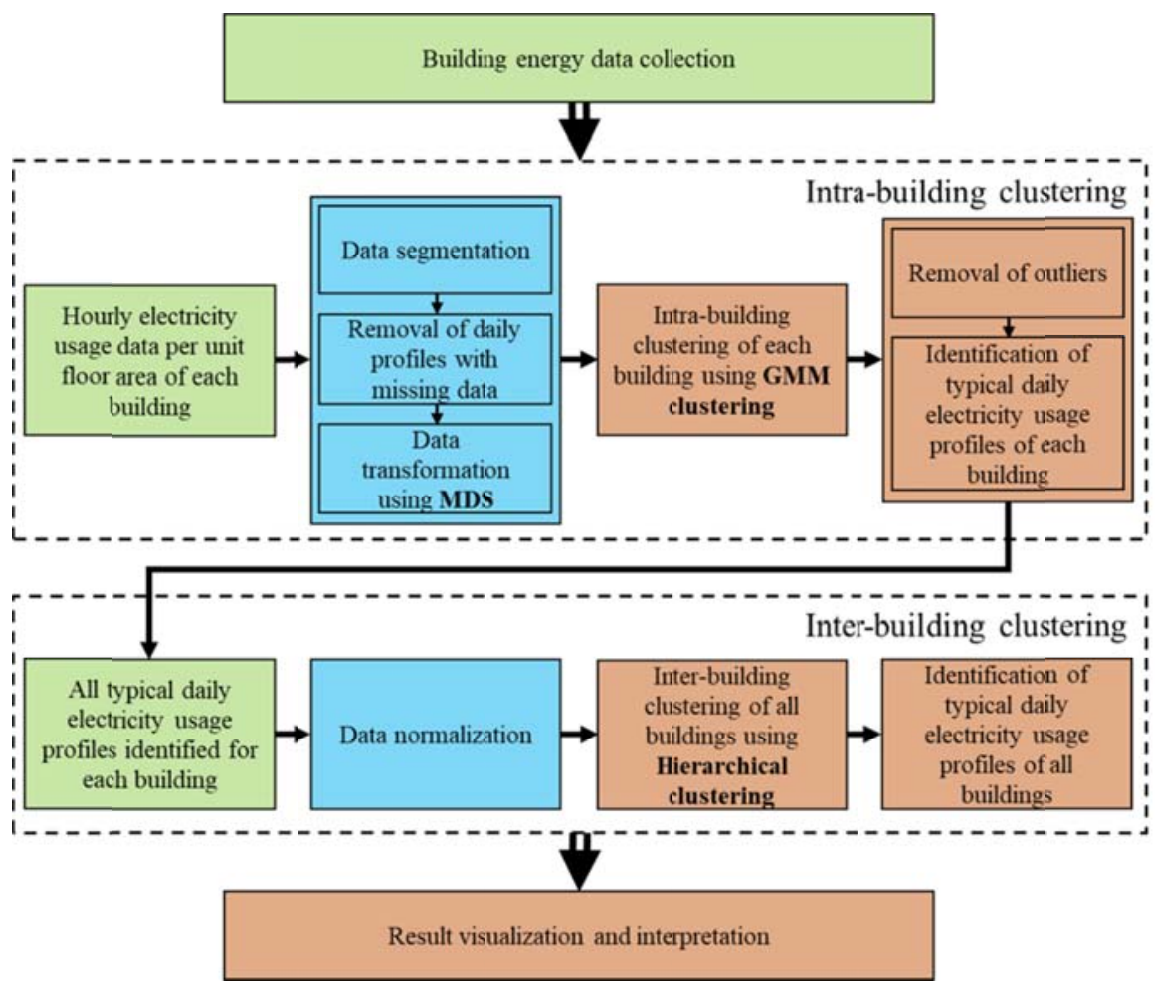

Fig. 1. Outline of the proposed strategy. 


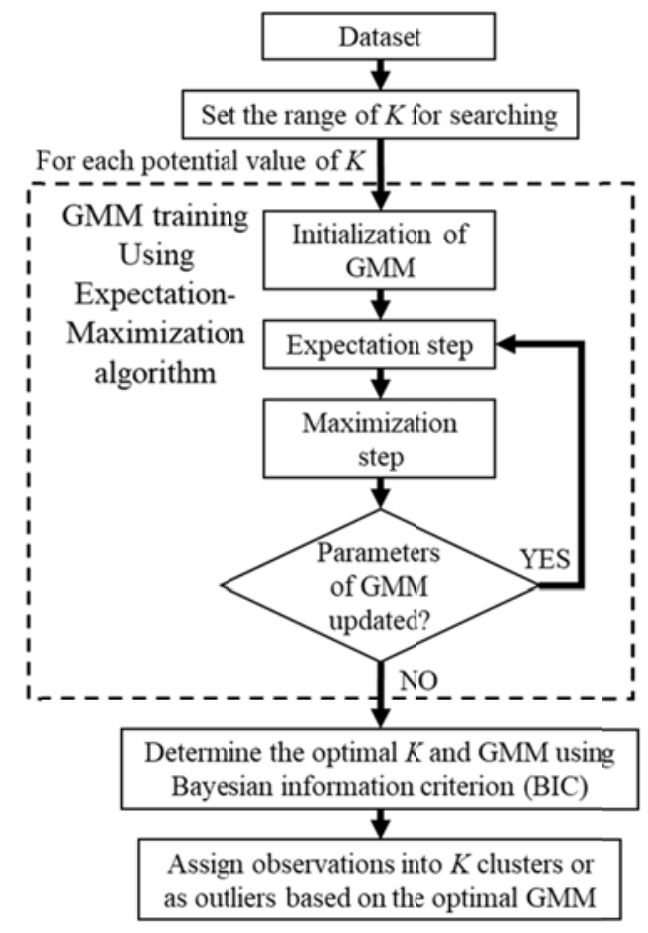

a) GMM-based clustering algorithm, where $K$ denotes the number of mixture components in a

\section{GMM}

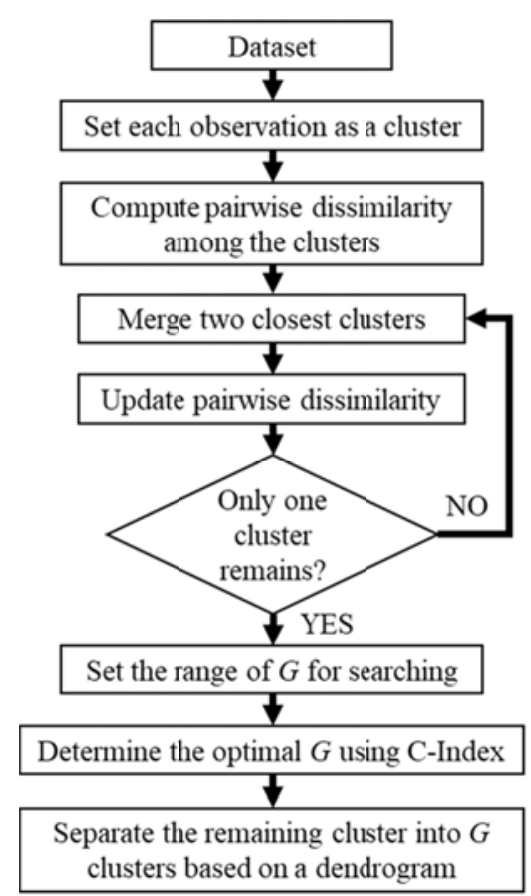

b) Agglomerative hierarchical clustering algorithm, where $G$ denotes the number of clusters

identified

Fig. 2. Flowcharts of the two clustering algorithms used in this study. 


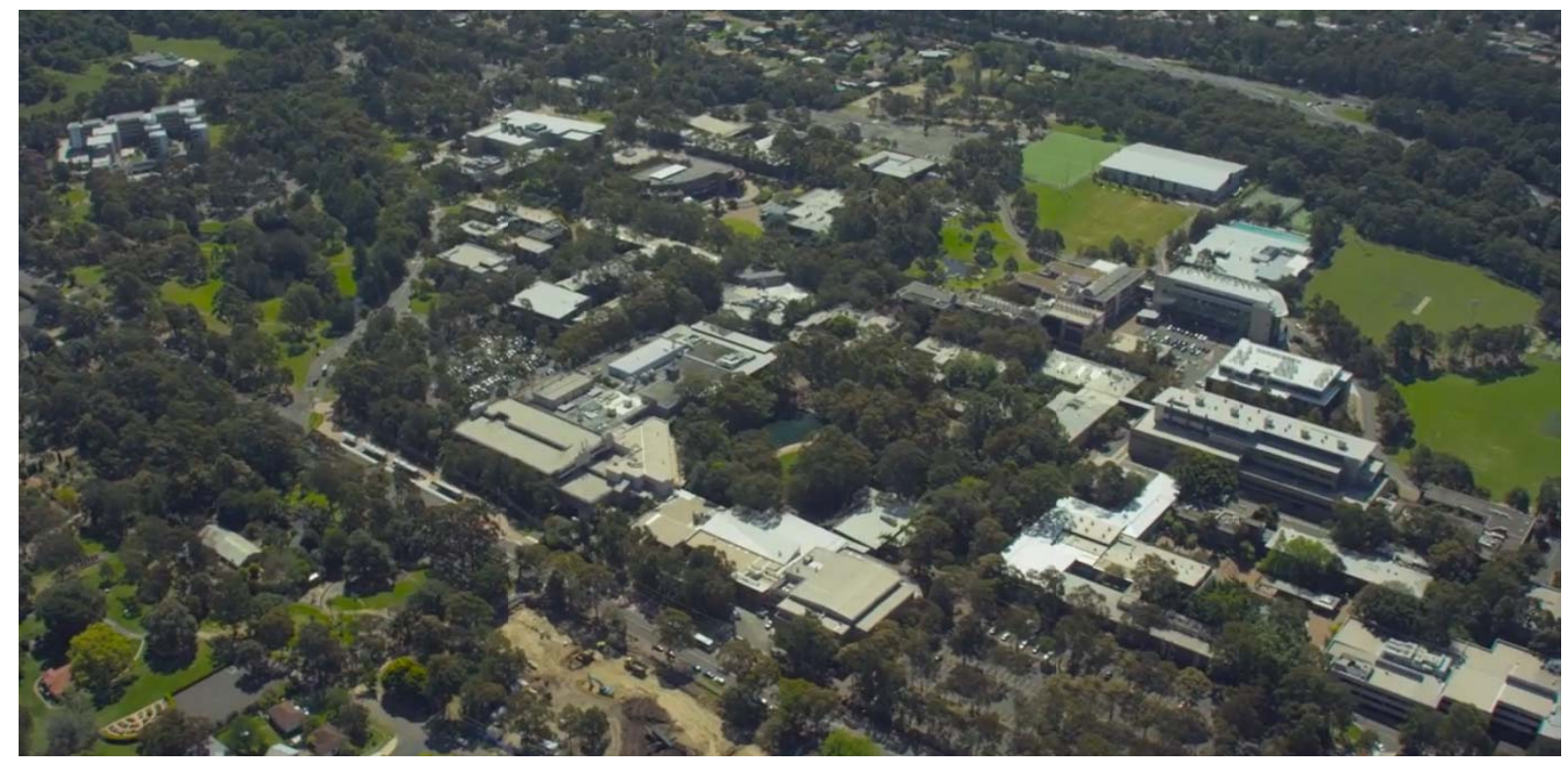

Fig. 3. Illustration of the university buildings concerned in this study.

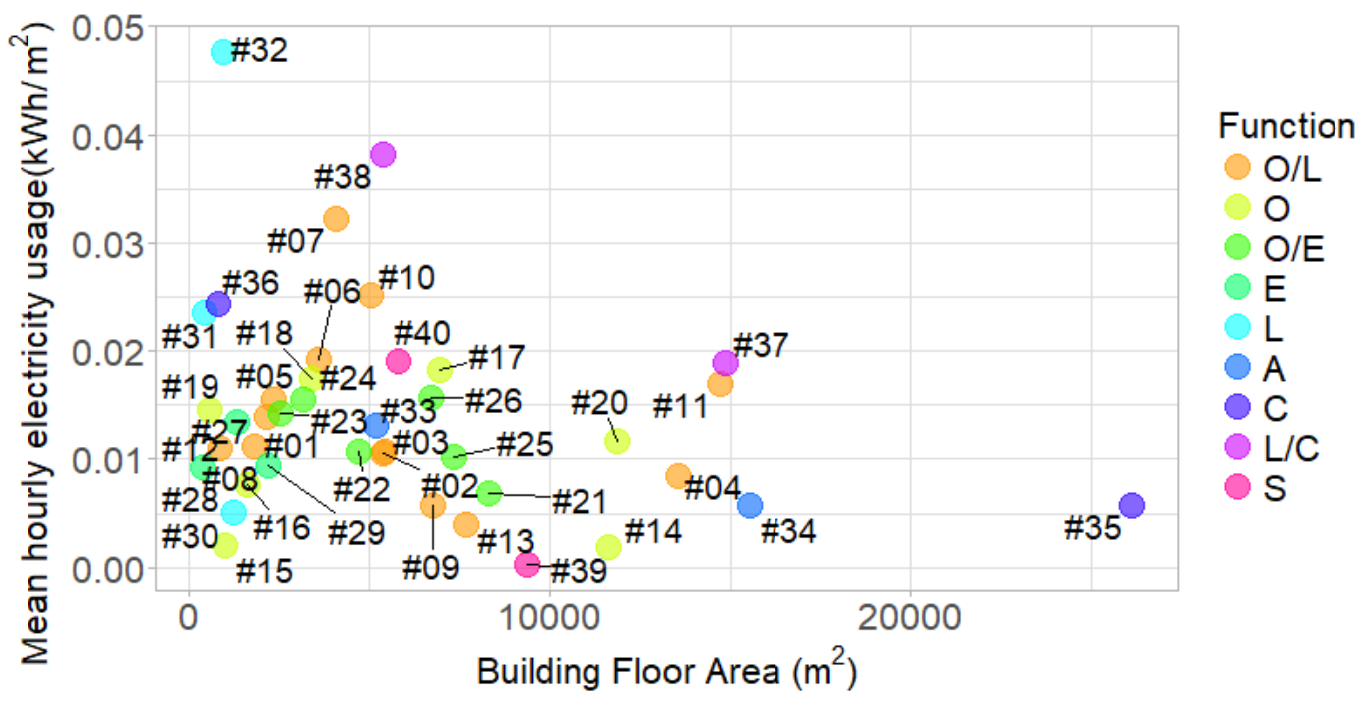

Fig. 4. Mean hourly electricity usage versus the floor area of 40 buildings.

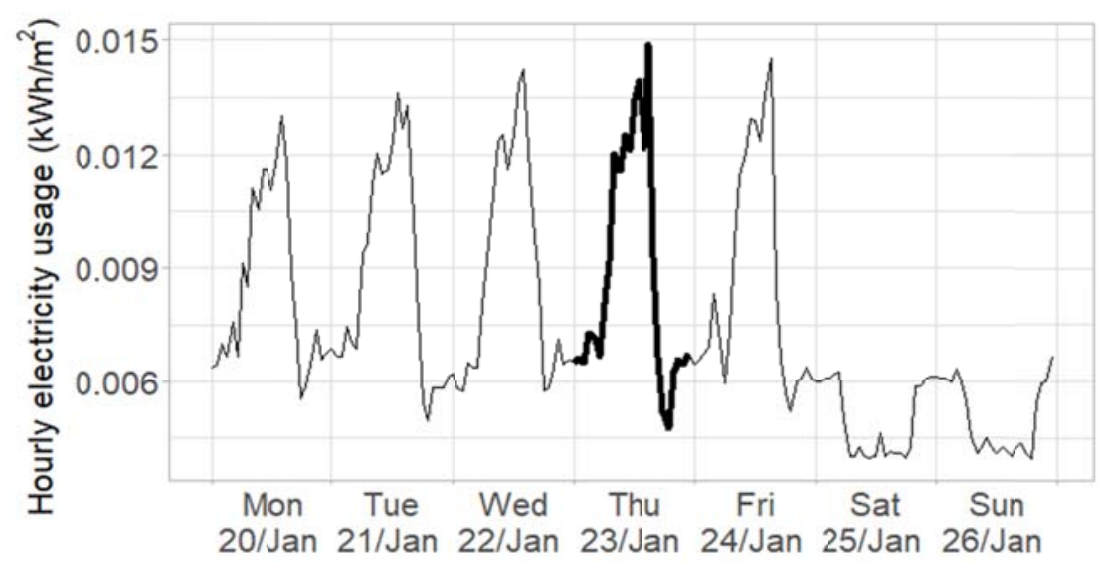


a) Daily electricity usage profiles before the data segmentation

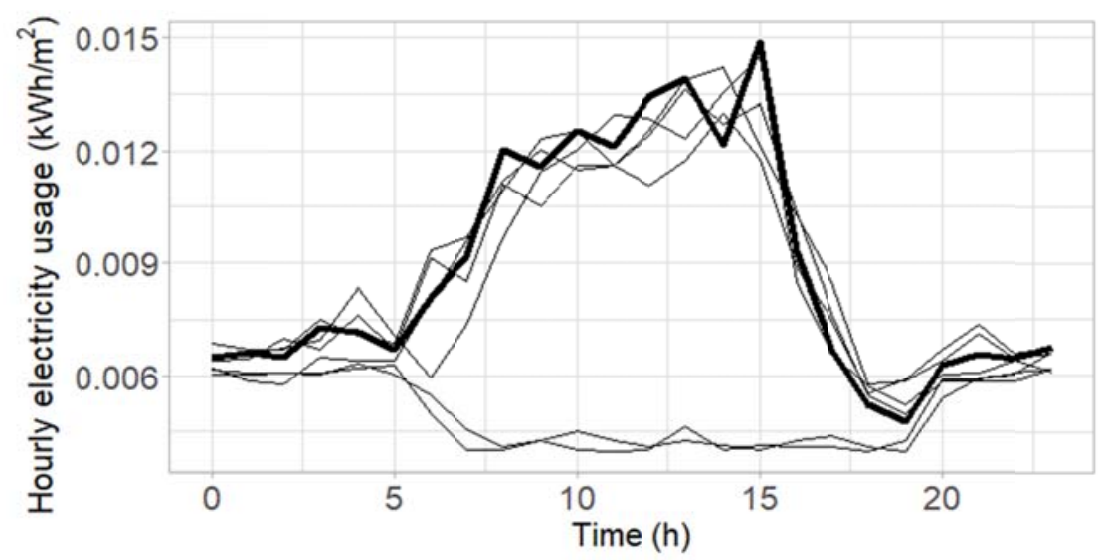

b) Daily electricity usage profiles after the data segmentation

Fig. 5. An illustration of the data segmentation.

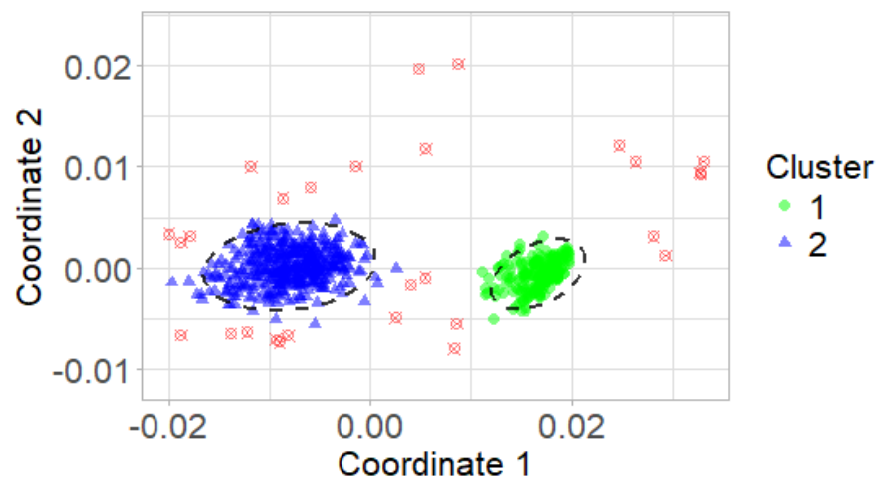

a) Building \#16

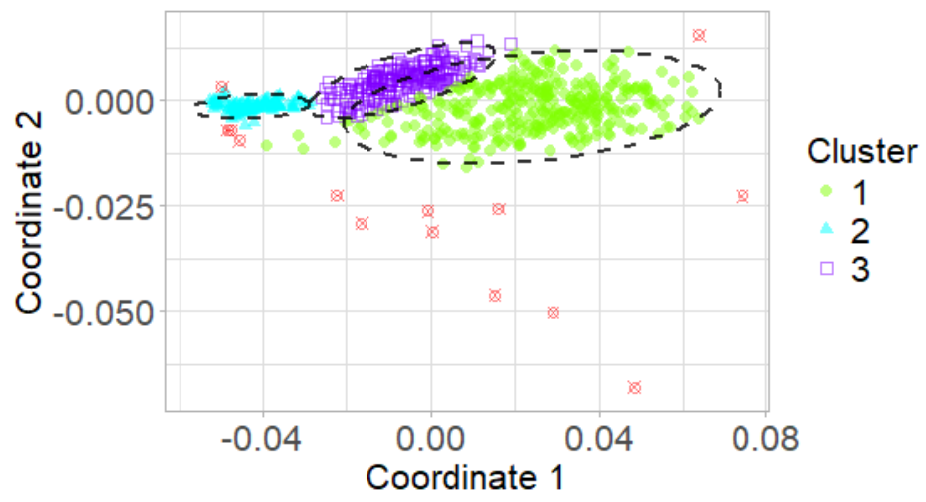

b) Building \#33 


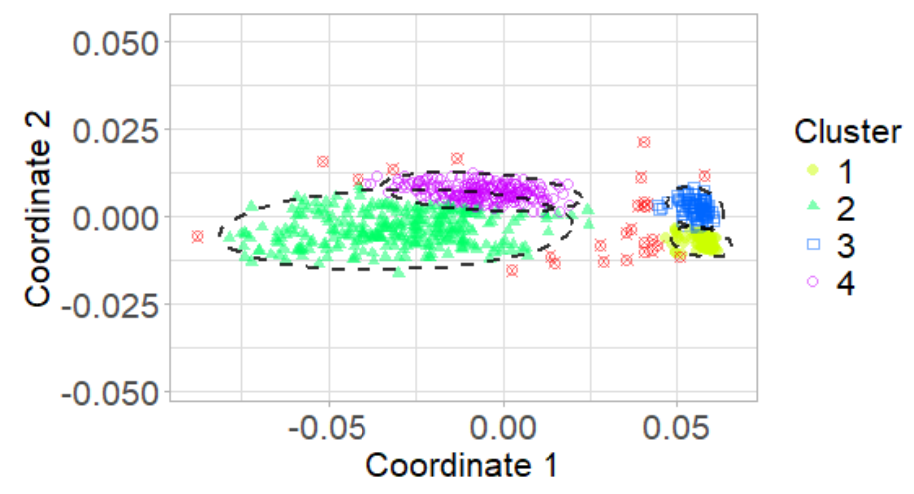

c) Building \#24

Fig. 6. A demonstration of the results from the GMM-based clustering.

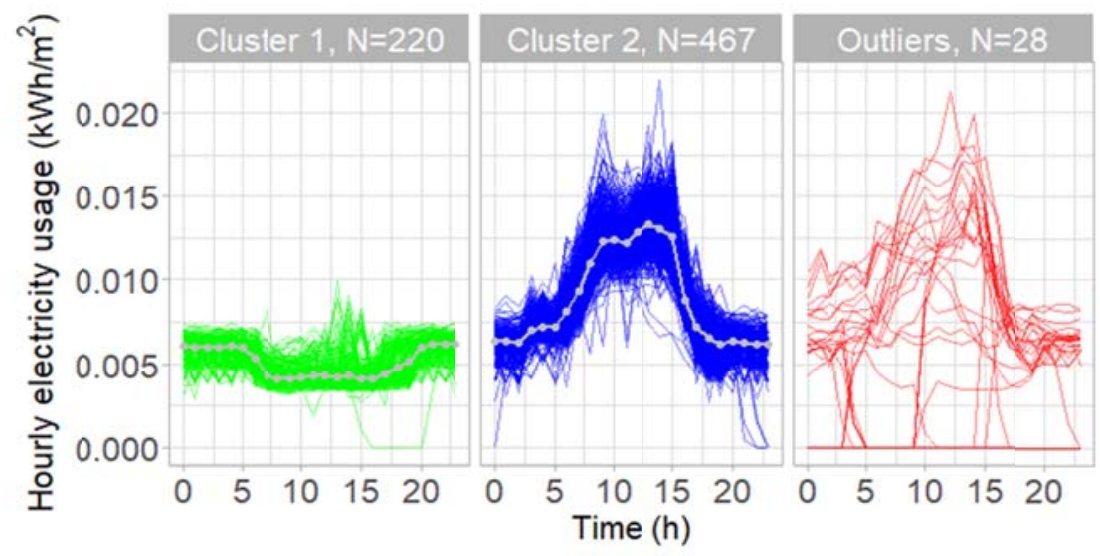

a) TDEU profiles and outliers

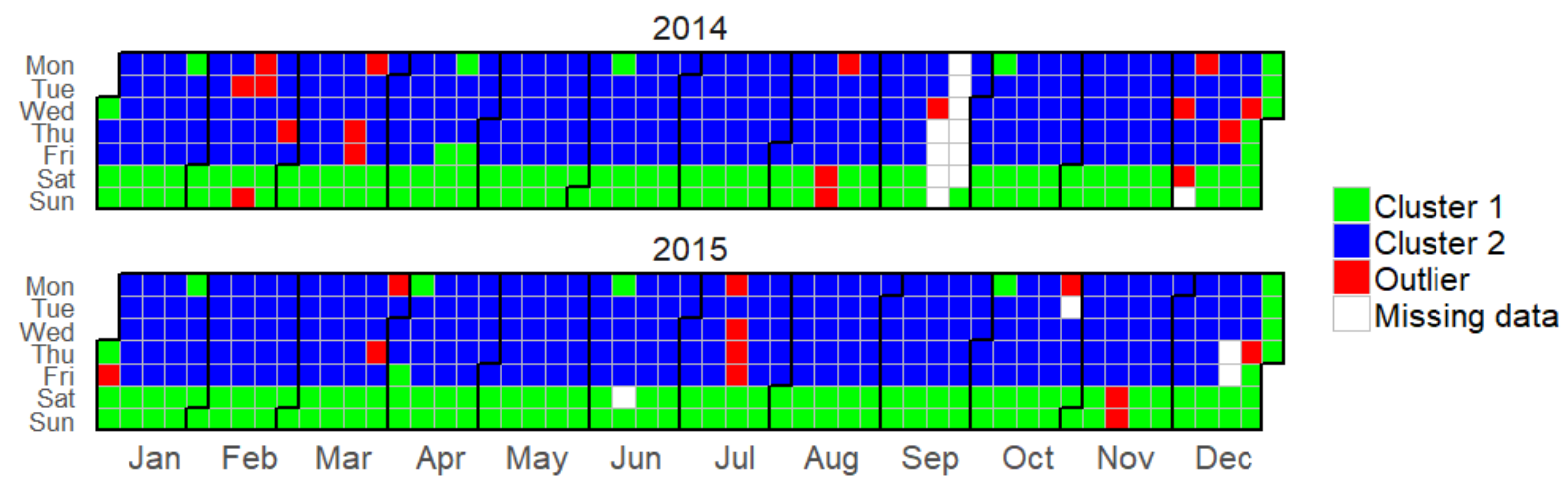

b) Distribution of the TDEU profiles

Fig. 7. Visualisation of the intra-building clustering result of Building \#16. 


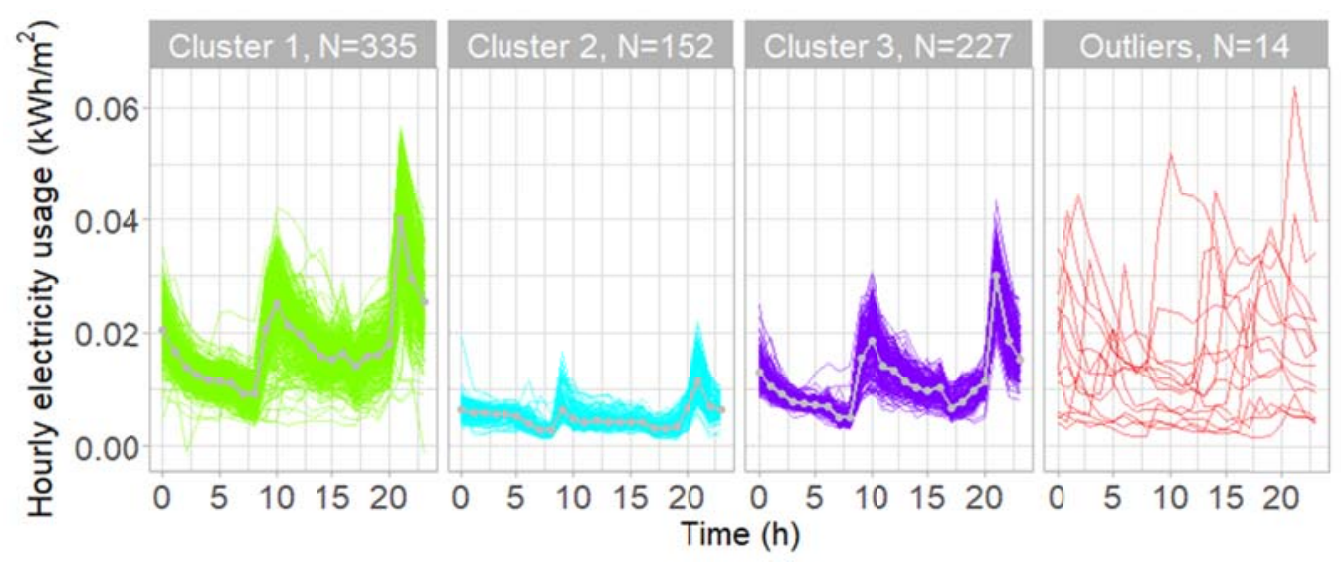

a) TDEU profiles and outliers

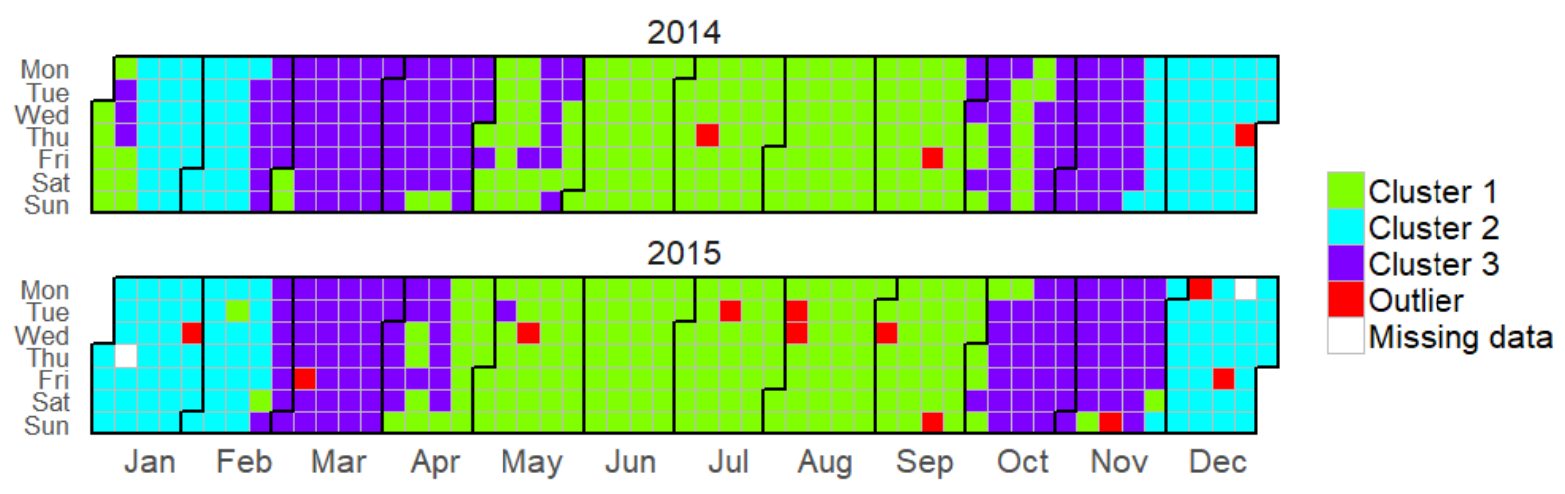

b) Distribution of the TDEU profiles

Fig. 8. Visualisation of the intra-building clustering result of Building \#33. 


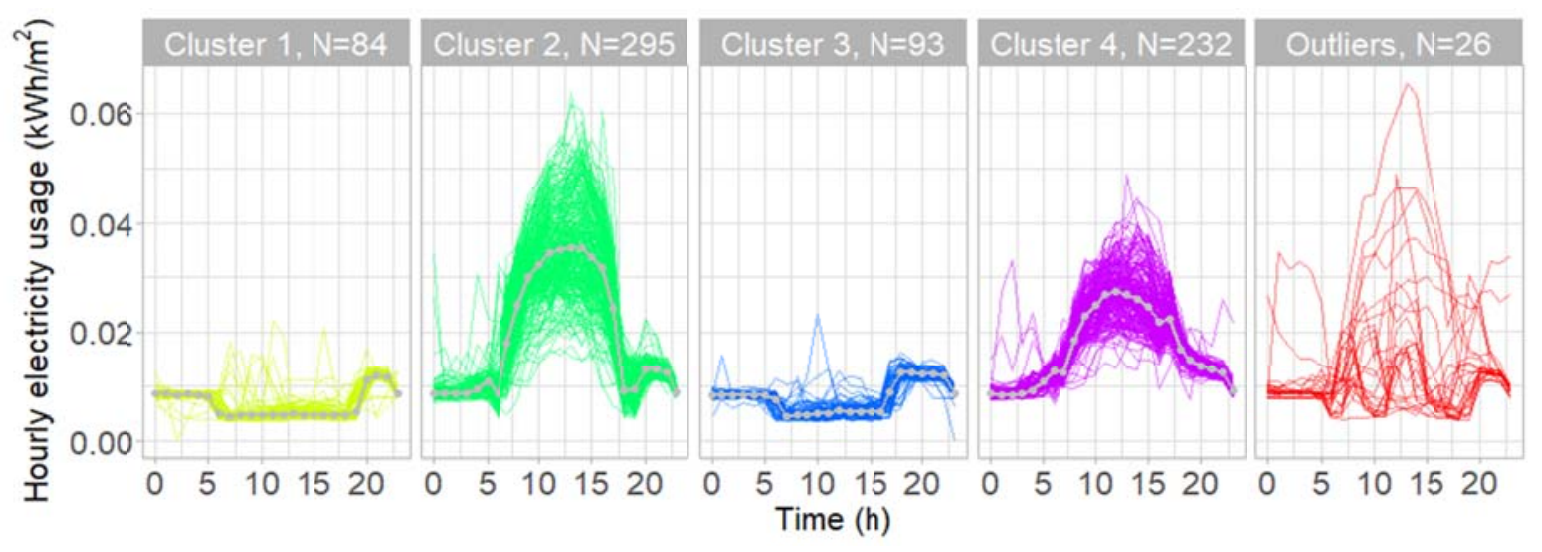

a) TDEU profiles and outliers

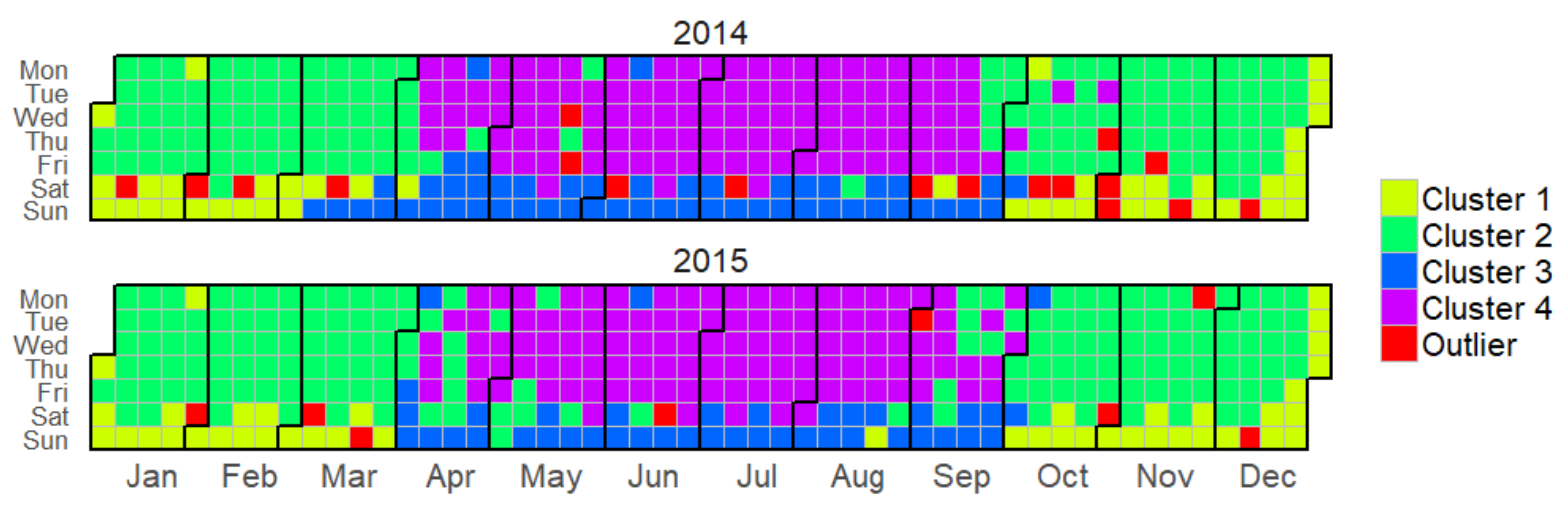

b) Distribution of the TDEU profiles

Fig. 9. Visualisation of the intra-building clustering result of Building \#24. 


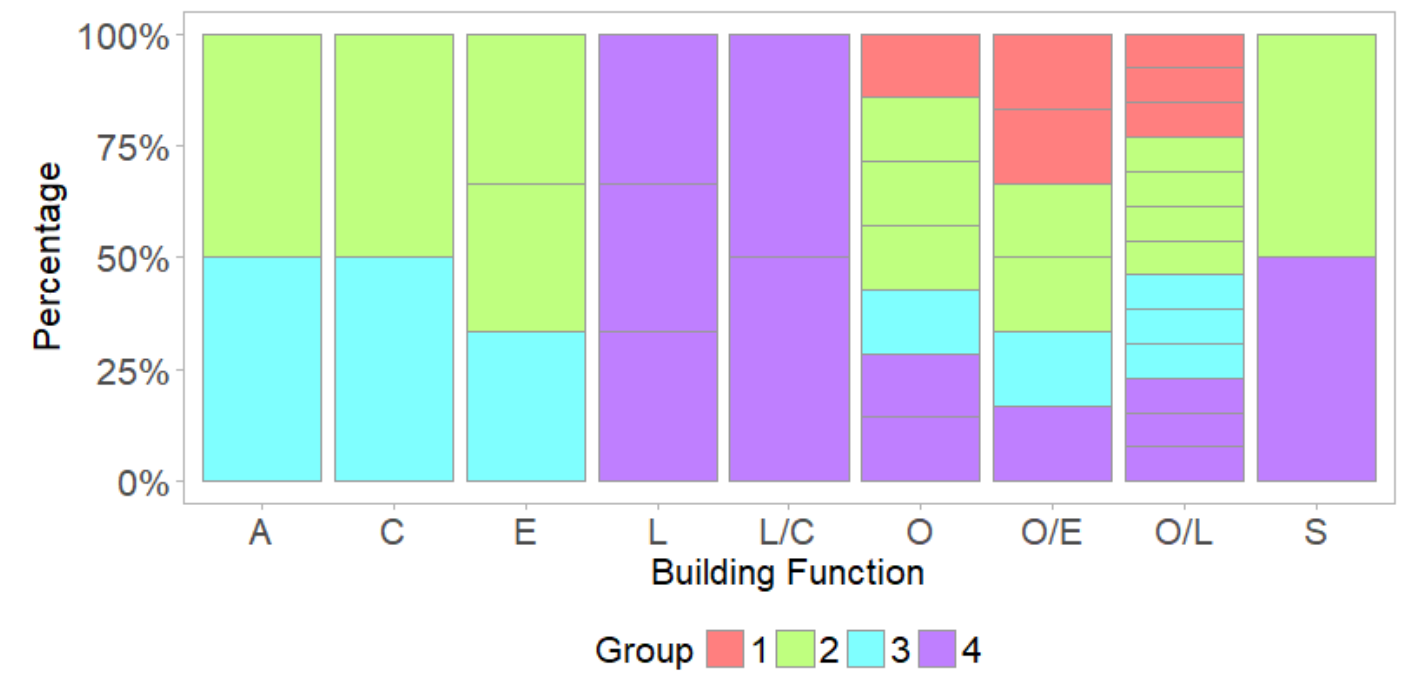

Fig. 10. Distribution of the buildings with different functions into the defined four groups.

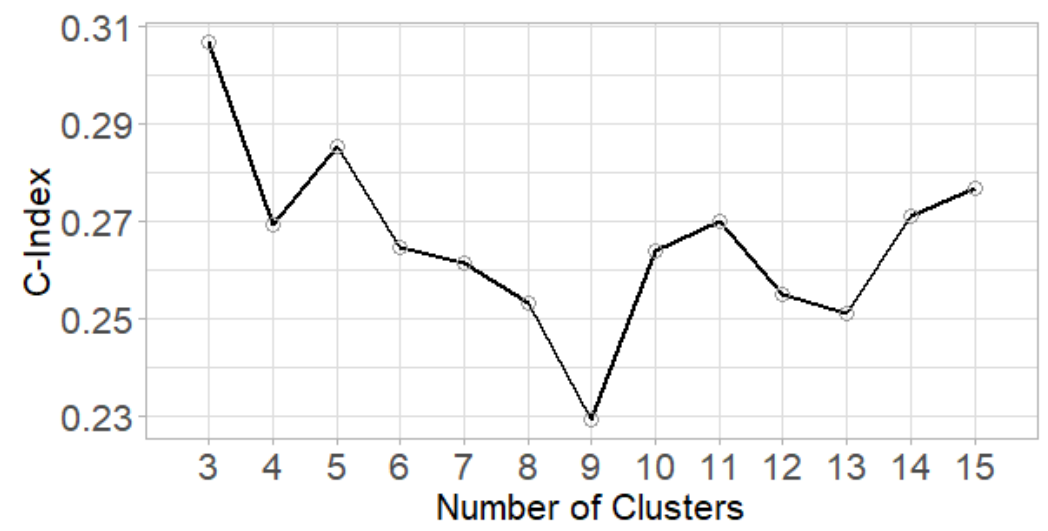

a) C-Index calculated for different numbers of the clusters

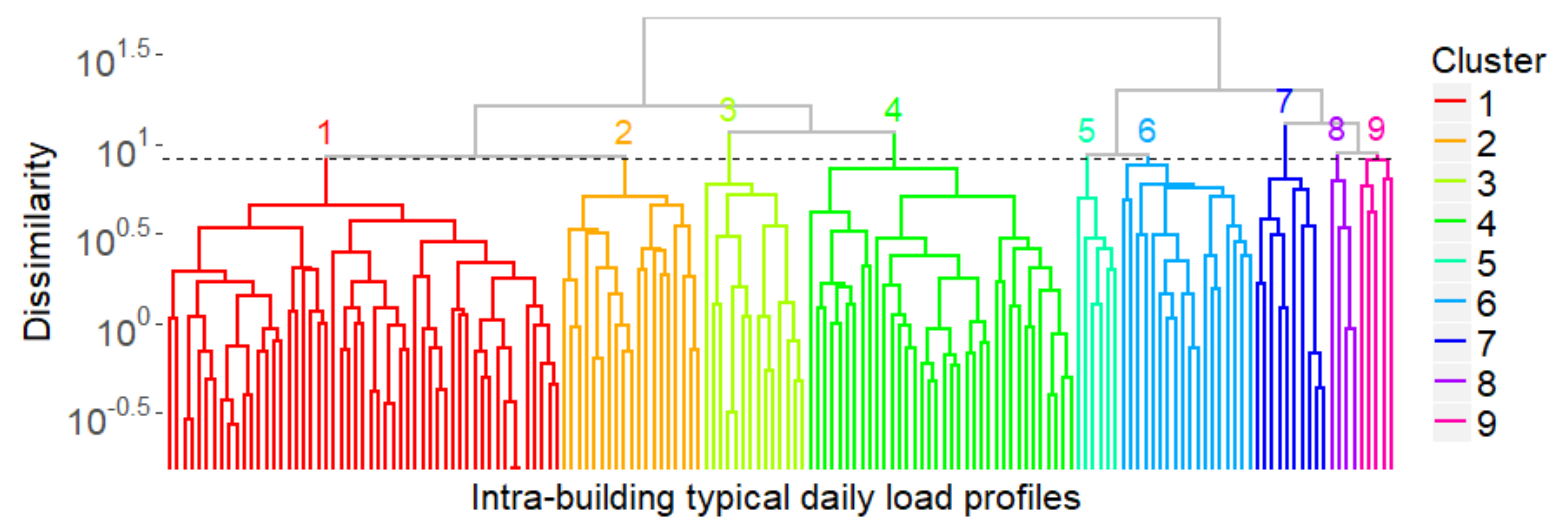

b) Dendrogram of the hierarchical clustering result

Fig. 11. The result of the agglomerative hierarchical clustering. 


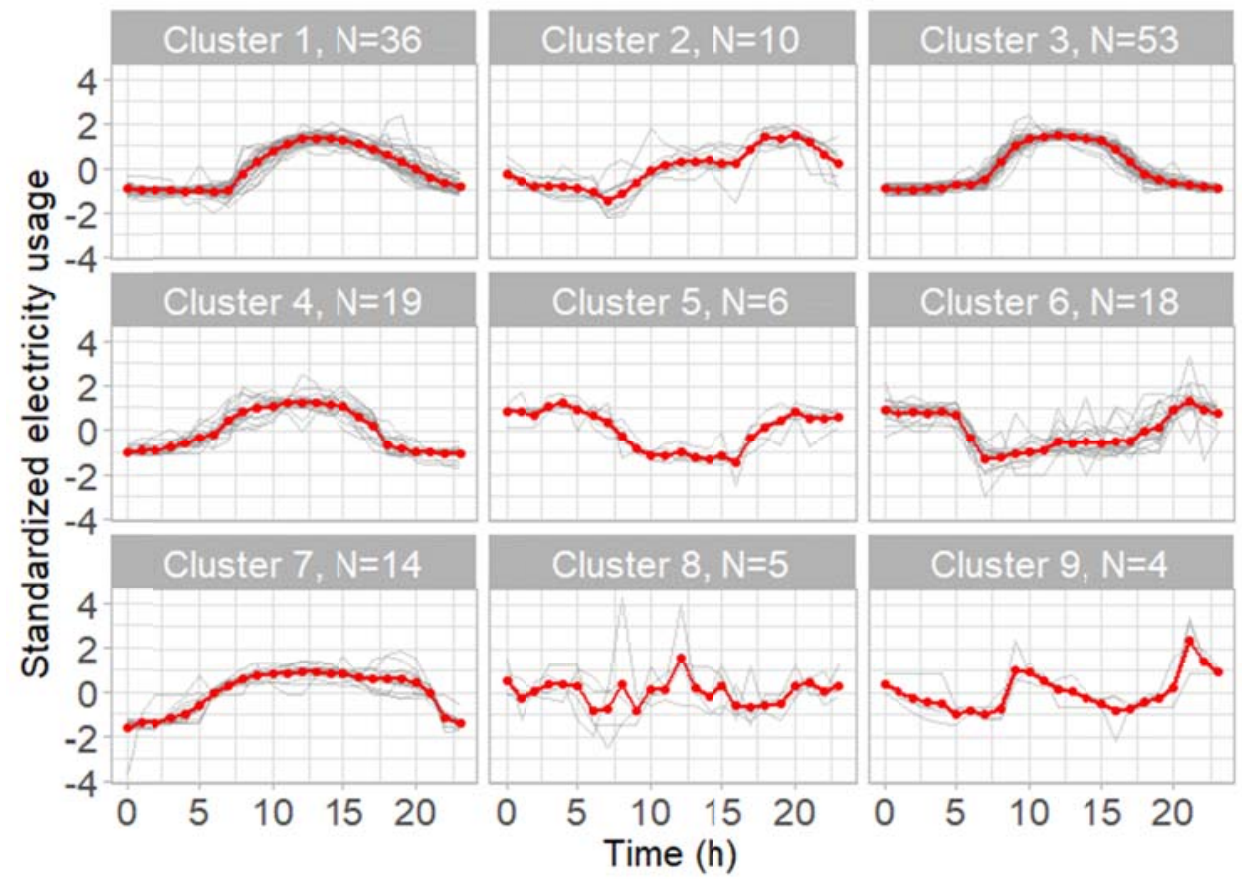

a) Average of TDEU profiles identified through intra-building clustering

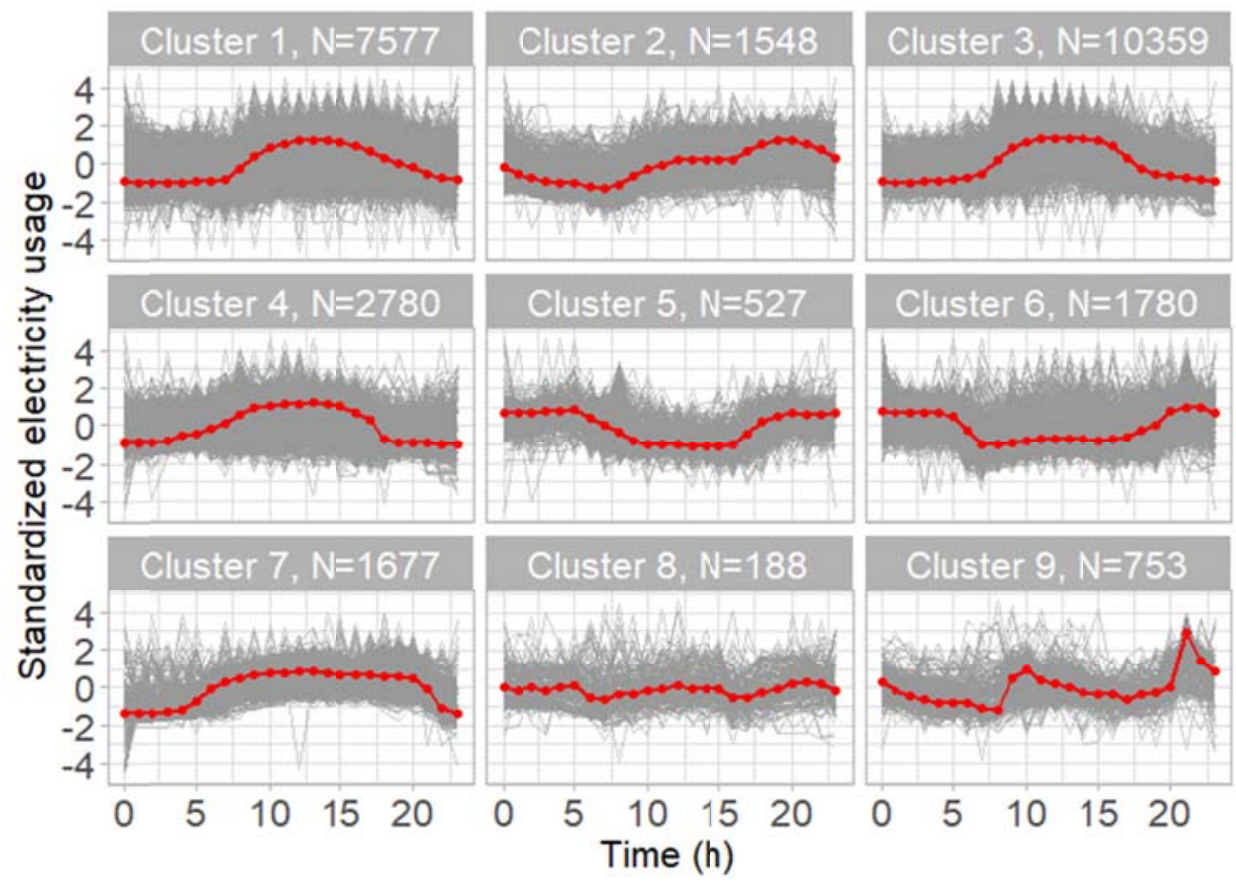

b) Average of original DEU profiles corresponding to the intra-building typical profiles

Fig. 12. TDEU profiles identified through hierarchical clustering for all 40 buildings. 


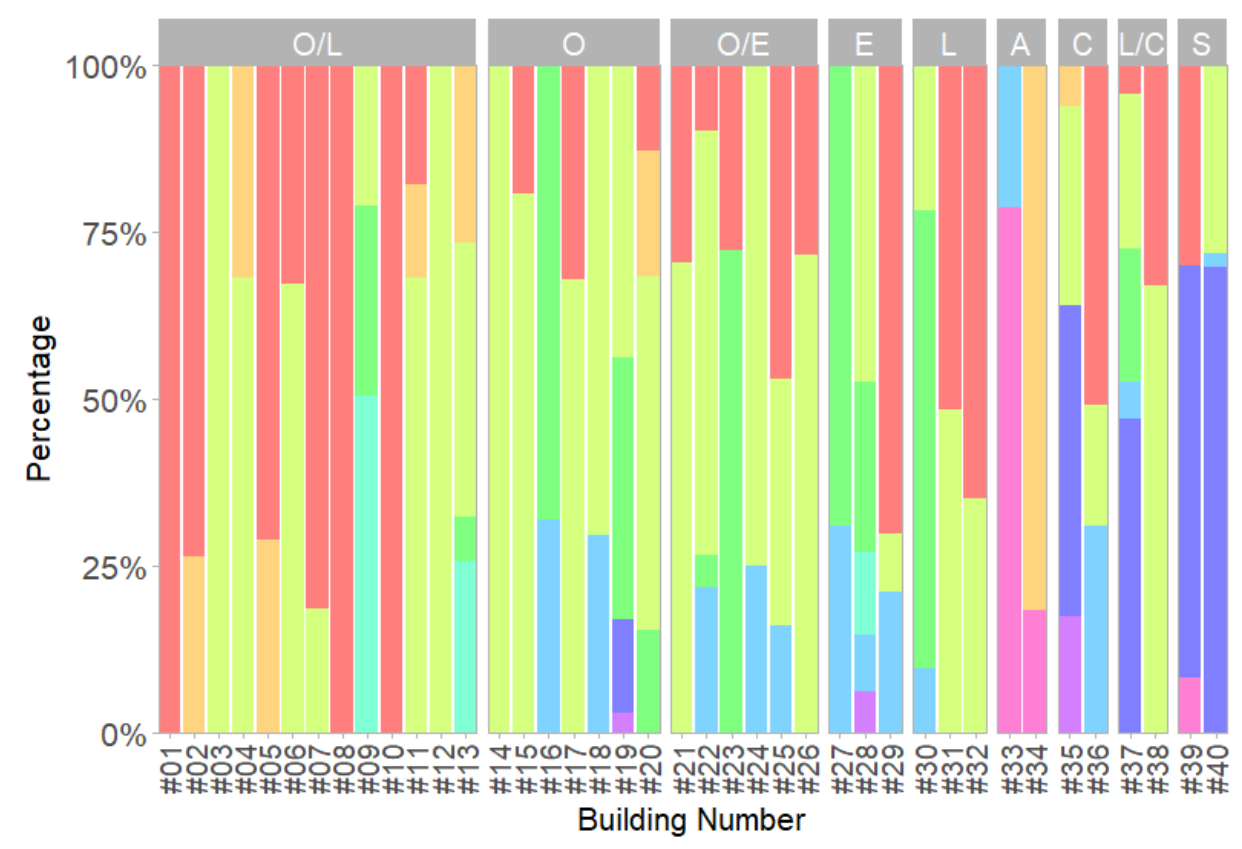

\begin{tabular}{ll|l|l|l|l|l|l|l|l} 
Cluster & 1 & 2 & 3 & 4 & 5 & 6 & 7 & 8 & 9
\end{tabular}

Fig. 13. The distribution of TDEU profiles of 40 buildings.

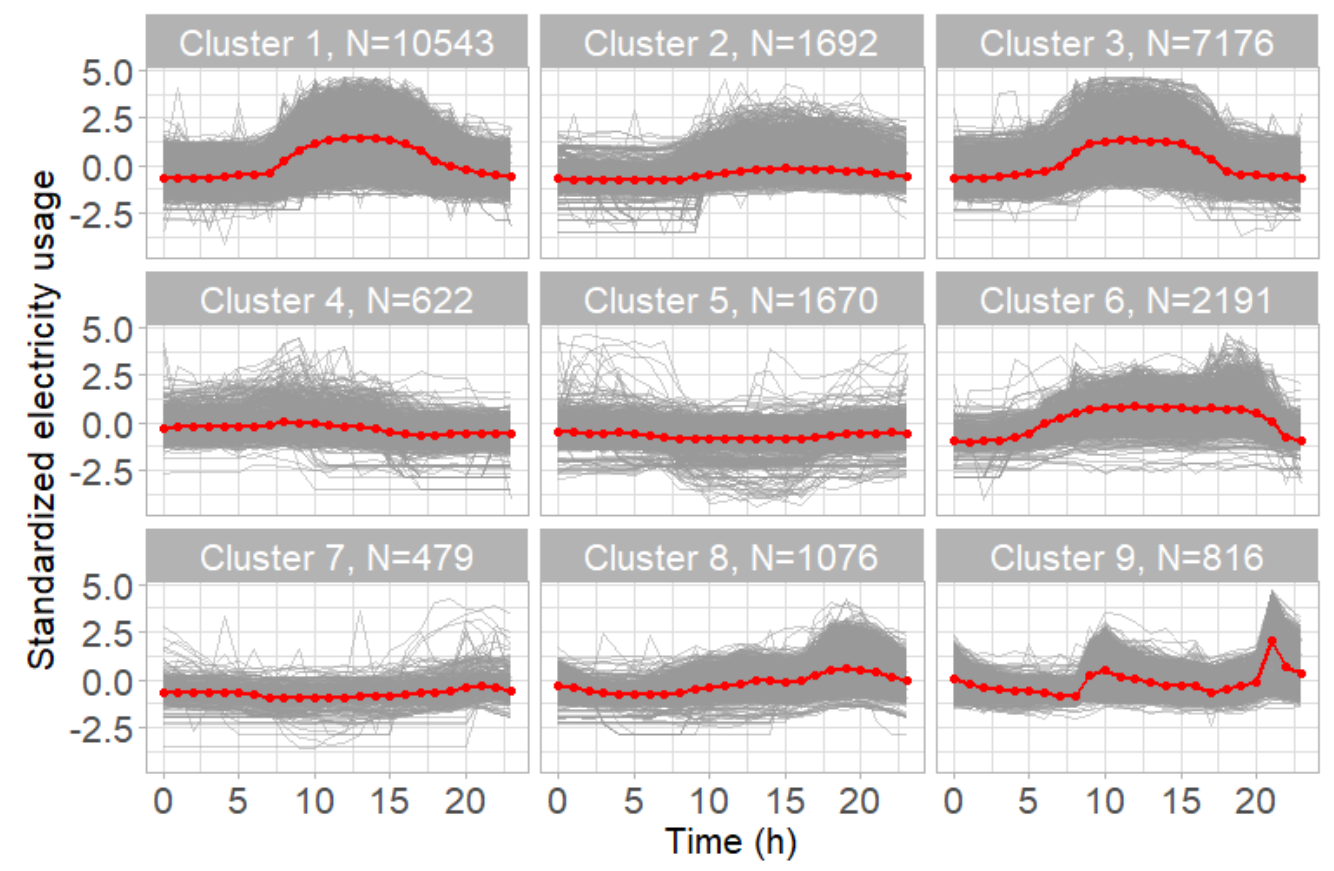

Fig. 14. Clustering result of the PAM-based strategy. 


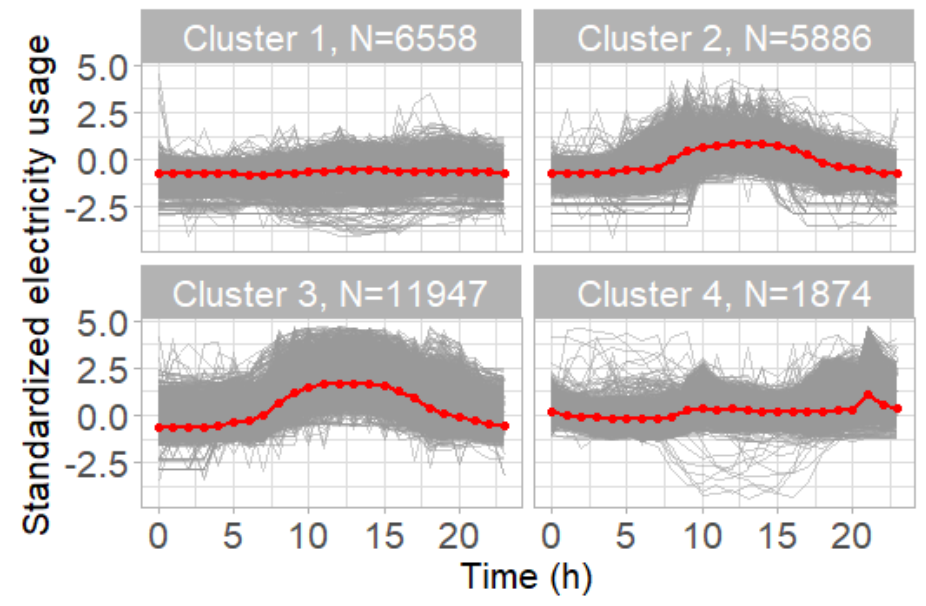

Fig. 15. Clustering result of the hierarchical clustering-based strategy. 